Portland State University

PDXScholar

\title{
Uniform Materials and the Multiplicative Decomposition of the Deformation Gradient in Finite Elasto-Plasticity
}

\author{
Vincenzo Ciancio \\ University of Messina \\ Marina Dolfin \\ University of Messina \\ Mauro Francaviglia \\ University of Torino \\ Serge Preston \\ Portland State University, serge@pdx.edu
}

Follow this and additional works at: https://pdxscholar.library.pdx.edu/mth_fac

Part of the Mathematics Commons

Let us know how access to this document benefits you.

\author{
Citation Details \\ Ciancio, V., Dolfin, M., Francaviglia, M., \& Preston, S. (2008). Uniform Materials and the Multiplicative \\ Decomposition of the Deformation Gradient in Finite Elasto-Plasticity. Journal Of Non-Equilibrium \\ Thermodynamics, 33(3), 199-234.
}

This Article is brought to you for free and open access. It has been accepted for inclusion in Mathematics and Statistics Faculty Publications and Presentations by an authorized administrator of PDXScholar. Please contact us if we can make this document more accessible: pdxscholar@pdx.edu. 


\title{
Uniform Materials and the Multiplicative Decomposition of the Deformation Gradient in Finite Elasto-Plasticity
}

\author{
Vincenzo Ciancio ${ }^{1}$, Marina Dolfin ${ }^{1}$, Mauro Francaviglia ${ }^{2}$, and \\ Serge Preston ${ }^{3, \star}$ \\ ${ }^{1}$ Department of Mathematics, University of Messina, I-98166 Messina, Italy \\ ${ }^{2}$ Department of Mathematics, University of Torino, I-10123 Torino, Italy \\ ${ }^{3}$ Department of Mathematics and Statistics, PO Box 751, Portland State \\ University, Portland, OR, 97207-0751, USA \\ ${ }^{\star}$ Corresponding author (serge@pdx.edu)
}

\begin{abstract}
In this work we analyze the relation between the multiplicative decomposition $\mathbf{F}=$ $\mathbf{F}^{e} \mathbf{F}^{p}$ of the deformation gradient as a product of the elastic and plastic factors and the theory of uniform materials. We prove that postulating such a decomposition is equivalent to having a uniform material model with two configurations - total $\phi$ and the inelastic $\phi_{1}$.

We introduce strain tensors characterizing different types of evolutions of the material and discuss the form of the internal energy and that of the dissipative potential. The evolution equations are obtained for the configurations $\left(\phi, \phi_{1}\right)$ and the material metric $\mathbf{g}$.

Finally, the dissipative inequality for the materials of this type is presented. It is shown that the conditions of positivity of the internal dissipation terms related to the processes of plastic and metric evolution provide the anisotropic yield criteria.
\end{abstract}

\section{Introduction}

The objective of this work is to investigate the relation between the geometrical theory of uniform materials and the multiplicative elasto-plastic decomposition of the deformation gradient of Bilby-Kröner-Lee (BKL decomposition) and Nemat-Nasser (see [1-4]). 
Such a relation was first studied in [5]. In particular, the relation between the inhomogeneity velocity gradient $\mathbf{L}_{P}$ (see below) and the plastic distortion rate $\overline{\mathbf{L}}=\overline{\mathbf{F}}^{p} \cdot\left(\overline{\mathbf{F}}^{p}\right)^{-1}$ was introduced. In this paper we study the geometrical form of the relation introduced in [5].

In Section 2 we introduce the basic concepts and review properties of uniform materials. In Section 3 a bijective correspondence between the BKL decompositions of the gradient of a configuration $\phi$ of an elasto-plastic solid and the triple $\left(\phi, \phi_{1}, P\right)$ is established. Here $P$ represents the uniform material structure and $\phi$ and $\phi_{1}$ are, respectively, total and inelastic (intermediate) material configurations.

In Section 4 we introduce the natural strain tensors measuring the relations between the Cauchy-Green deformation tensors $C(\phi)$ and $C\left(\phi_{1}\right)$ and the material metric $\mathbf{g}$ induced by the uniform structure $P$. In the same section, the combinations of these tensors, material metric and its curvature characteristic independent of the decomposition of plastic deformation gradient $F^{p}=$ $\phi_{1 *} \circ D$ are determined and the strain rate tensors are introduced.

In Section 5 the form of the internal energy $u$ depending on variables $\left(\phi, \phi_{1}, \mathbf{g}\right)$ and their derivatives is postulated and the dissipative potential $\mathcal{D}$ is introduced. We also formulate the system of equations describing evolution of dynamical variables $\left(\phi, \phi_{1}, \mathbf{g}\right)$. In the same section, different stress tensors present in our scheme are defined and relations between them are discussed.

In Section 6 we write down the dissipative inequality for the suggested scheme and separate the terms corresponding to the internal dissipation related to the processes of integrable inelastic and uniform structure evolutions. We show that the conditions of positivity of the corresponding terms in dissipative inequality provide the anisotropic yield criteria for initiating the corresponding processes.

Another form of a relation between the finite elasto-plasticity based on the multiplicative decomposition and the uniformity structures using the secondorder connection was suggested by Cleja-Tigoui, see [6].

\section{Uniform materials: material connections and material metrics}

Uniform materials entered the scene of material science in about 1952 when Kondo introduced the material connection and the material metric as the tools to model properties of materials. Later developments in the works by Kondo, Bilby and his collaborators, Kroner, Noll ([7]) and Wang (see [8, 9]) establish the basis of this theory. In the works from 1980 to present by 
Elzanowski, Epstein, de Leon, Maugin, different aspects of this theory were further developed: models of higher grade uniform materials, dynamics of material properties, thermodynamical properties of such materials, role of Eshelby stress tensor, geometry of functionally graduate material, etc.

In this section we present the basic geometrical structures of the theory of uniform materials that will be used in later parts of the paper. Our presentation is based on [5,10-13].

\subsection{Material and physical spaces}

A material body (material manifold) is usually represented by a connected 3dimensional smooth oriented manifold $M$ with a piecewise smooth boundary $\partial M$. Constructions of this paper are local, so it is sufficient to consider $M$ as a connected open domain in $\mathbb{R}^{3}$ with local coordinates $X^{I}, I=1,2,3$.

As the physical space our body is placed in, we consider the 3-dimensional Euclidean vector space $\left(E^{3}, \mathbf{h}\right), \mathbf{h}$ being the (flat) Euclidean metric. We introduce global Cartesian coordinates $x^{i}$ in $\mathbb{R}^{3}$. In these coordinates the metric $\mathbf{h}$ takes the form $\mathbf{h}=h_{i j} d x^{i} d x^{j}$.

We will also use the concept of "archetype" (see [10] or [11], Sec. 1.2.), a 3-dimensional vector space $V$ endowed with a standard Euclidean metric and the orthonormal basis $\mathbf{e}_{0}=\left\{e_{i}, i=1,2,3\right\}$. For convenience, we identify the "archetype" space $V$ with the tangent space at the origin $O$ of the physical space: $V=T_{O}\left(\mathbb{R}^{3}\right)$ and its metric with the metric $\mathbf{h}$ at the origin.

\subsection{Configurations and the Cauchy metric}

Configuration of the body $M$ is a (diffeomorphic) embedding $\phi: M \rightarrow E^{3}$ into the physical space $E^{3}$; see [15], Ch. 1 . To each configuration $\phi$, there corresponds the deformation gradient - the mapping from the tangent space $T_{X}(M)$ at the point $X \in M$ to the tangent space $T_{\phi(X)}\left(E^{3}\right)$ at the point $\phi(X) \in E^{3}$,

$$
\mathbf{F}(X)=\phi_{* X}: T_{X}(M) \rightarrow T_{\phi(X)}\left(E^{3}\right),
$$

given, in coordinates $X^{A}, x^{i}$, by the matrix of partial derivatives

$$
\mathbf{F}(X)_{I}^{i}=\phi_{, I}^{i} \text {. }
$$

Here and below we will use the notation $\phi_{, I}^{i}=\frac{\partial \phi^{i}}{\partial X^{I}}(X)$ for the partial derivatives of configuration components $\phi^{i}(X)$. 
To a configuration $\phi(X)$ there corresponds the right Cauchy-Green deformation tensor - the flat metric $\mathbf{C}(\phi)=\phi^{*} \mathbf{h}$ in $M$ obtained as the pullback of Euclidian metric $h$ in physical space by the configuration mapping $\phi$. In coordinates $\left(X^{I}\right)$ tensor $C(\phi)$ has the form

$$
C(\phi)_{I J}=h_{i j} \phi_{I}^{i} \phi_{J}^{j}
$$

We will fix a specific configuration $\phi_{o}$ and call it the reference configuration. Usually it presents the state of the material body that is free from loads and stresses (see [14], p. 15 or [16], p. 48), although it might happen that such a configuration does not exist and one has to choose a reference configuration differently. The body $M$ is often identified with its image under the embedding $\phi_{o}$.

To the reference configuration $\phi_{o}$ there corresponds its Cauchy-Green tensor called the reference metric in $M$,

$$
\mathbf{g}_{o}=\mathbf{C}\left(\phi_{o}\right), g_{o I J}=h_{i j} \phi_{o, I}^{i} \phi_{o, J}^{j},
$$

and the corresponding reference volume form $v_{o}(X)=\sqrt{\left|g_{o}\right|} d X^{1} \wedge \ldots \wedge d X^{n}$. Using the mapping inverse to the reference configuration $\phi: M \rightarrow E^{3}$, one can define the frame $\mathbf{p}_{o}$ in $M$ by the rule ${ }^{1}$

$$
\mathbf{p}_{o}(X)=\phi_{o, * X}^{-1}\left(\mathbf{e}_{0}\right),\left(p_{o}\right)_{i}=\frac{\partial \phi_{o}^{-1 I}}{\partial x^{i}} \frac{\partial}{\partial X^{I}}, i=1,2,3 .
$$

From now on we assume that the coordinates $X^{I}$ are introduced in the material manifold $M$ using the reference configuration, i.e., $X^{I}(X)=\phi_{o}^{I}(X)$. Then the vectors of the frame $\mathbf{p}_{o}$ take the form $\left(p_{o}\right)_{I}=\frac{\partial}{\partial X^{I}}, I=1,2,3$.

Finally we define a history of deformation as a time parameterized family of smooth configurations: $\phi(t, X): M \times \mathbb{R} \rightarrow E^{3}$.

\subsection{Uniform materials, I}

Recall $[7,8]$ that a material is called hyperelastic if its constitutive response (to a loading condition) at any configuration $\phi$ is completely characterized by two scalar functions:

\footnotetext{
${ }^{1}$ Here and below for a differentiable mapping $\psi: M \rightarrow N$ between manifolds $M$ and $N$ we denote by $\psi_{* X}: T_{X}(M) \rightarrow T_{\psi(X)}(N)$ the linear mapping of tangent spaces at a point $X \in M$. In coordinates $\left(X^{I}, x^{i}\right)$ mapping $\psi_{* X}$ is given by the matrix $F_{I}^{i}=\phi_{, I}^{i}$. Corresponding mapping of the tangent bundles will be denoted by $\psi_{*}: \psi_{*}: T(M) \rightarrow T(N)$; see [15], Ch. 1 .
} 
1. The elastic energy density function (per unit of reference volume $v_{o}$ ) $W(X, \mathbf{F}(X))$ depending on a material point $X \in M$ and the deformation gradient $\mathbf{F}(X)$ at this point; and

2. The mass density function $\rho_{\text {ref }}(X)>0$ in the reference configuration $\phi_{o}$.

Next, we introduce the basic notion of a uniform material (body). Intuitively speaking, a uniform body is one that is made of the same material at all its points. The property of uniformity is characterized in terms of a parallelism $K_{X}^{Y}$ in the body $M[8,10,16]$. More specifically, a hyperelastic material body $(M, W)$ is called uniform if for any two material points $X, Y$ there exists a linear isomorphism $K_{X}^{Y}: T_{X}(M) \rightarrow T_{Y}(M)$ between tangent spaces at these points such that

$$
K_{X}^{Y *}\left(W(Y, \mathbf{F}(Y)) d v_{0}(Y)\right)=W\left(X, F(Y) \circ K_{X}^{Y}\right) d v_{0}(X)
$$

for all values of deformation gradients $\mathbf{F}(Y)$ at $Y$. Here $K_{X}^{Y *}$ is the pullback of the n-form of energy density by the mapping $K_{X}^{Y}$.

We now introduce the scalar factor $\lambda_{X}^{Y}$, characterizing the behavior of the reference volume form under the parallelism $K_{X}^{Y}: K_{X}^{Y *} v_{o}(Y)=\lambda_{X}^{Y} v_{o}(X)$. Then, in terms of the energy density function $W$, condition (3) takes the form

$$
\lambda_{X}^{Y} W(Y, \mathbf{F}(Y))=W\left(X, \mathbf{F}(Y) \circ K_{X}^{Y}\right)
$$

for all points $X, Y$ in $M$ and for all values of deformation gradient $\mathbf{F}(Y)$ at the point $Y$.

\subsection{Material connections}

The localization of the definition of uniform materials given above leads to the introduction of a linear connection (material connection) $\omega$ in $M$ having vanishing curvature (an absolute parallelism; see [17], Ch. 3, Sec. 2. Having such a connection available, the mappings $K_{X}^{Y}$ are defined by the parallel translation defined by connection $\omega$ from the point $X$ to the point $Y$ along any curve connecting $X$ and $Y$ (result of such translation is independent of the choice of a curve due to the vanishing of the curvature). The torsion tensor $T$ of connection $\omega$ provides the measure of nonhomogeneity of the material; see $[10,18]$.

It is known (see [17], Ch. 2) that in a simply connected body $M$, which admits a global tangent frame, a zero curvature connection is determined by a choice of a global tangent frame parallel with respect to the connection $\omega$ :

$$
\mathbf{p}(X)=\left\{\mathbf{p}_{k}=p_{k}^{I}(X) \partial_{X^{I}}, k=1, \ldots, 3, \nabla^{\omega} \mathbf{p}_{k}=0\right\} .
$$


Remark 1 A choice of such a frame is unique up to the (natural) right action of the group $G L(n, R)$ on the tangent frames and the left action of the symmetry gauge group $G^{M}$ of the connection $\omega$ (see $\left.[11,13]\right)$.

A global frame $\mathbf{p}$ may also be defined by the uniformity mapping smoothly depending on the point $X$ :

$$
P_{X}: V \rightarrow T_{X}(M), P_{X}\left(\mathbf{e}_{i}\right)=\left(P_{X}\right)_{i}^{I} \partial_{X^{I}}, i=1,2,3 .
$$

Mapping $P_{X}$ defines the linear isomorphism of the archetype space $V$ with the tangent space at each point $X \in M$. Section $\mathbf{p}$ and the uniformity map $P$ are related by

$$
\mathbf{p}(X)=P_{X}\left(\mathbf{e}_{0}\right) \Leftrightarrow p_{I}(X)=P_{I}^{J} \frac{\partial}{\partial X^{J}}
$$

Parallel translation $K_{X}^{Y}$ defined by the connection $\omega$ can be written in terms of the uniformity mapping as the composition

$$
K_{X}^{Y}=P_{Y} \circ P_{X}^{-1}
$$

Using the reference frame $\mathbf{p}_{o}$ (see above) and the frame $\left\{\mathbf{e}_{i}\right\}$ in the space $V$, one can associate to a material frame $\mathbf{p}$ two other geometrical objects:

1. A smooth mapping $k: M \rightarrow G L(V), X \rightarrow k(X)$ (an element of the gauge group $G L(V)^{M}$ ) such that for all $X \in M$

$$
\mathbf{p}_{J}(X)=\mathbf{p}_{o}(X) \cdot k(X) \Leftrightarrow p_{J}^{I}(X)=p_{0}^{L} k(X)_{L}^{I}, I, J=1,2,3,
$$

here $G L(V)$ is the group of invertible linear transformations of the archetype space $V$;

2. A nondegenerate $(1,1)$-tensor field $D_{J}^{I}(X)$ such that

$$
\begin{aligned}
\mathbf{D}(X) \mathbf{p}_{o}(X) & =\mathbf{p}(X), \quad \text { i.e. } \\
p_{i}^{I}(X) & =D(X)_{J}^{I}\left(p_{0}\right)_{i}^{J}(X)=D_{i}^{I}(X), i, I=1, \ldots, 3,
\end{aligned}
$$

last equality being true due to $\left(p_{0}\right)_{i}^{I}(X)=\delta_{i}^{I}$.

Nondegeneracy of the (1,1)-tensor $\mathbf{D}(X)$ means that $\mathbf{D}(X) \in G L\left(T_{X}(M)\right)$.

Using the relation between the frame $\mathbf{p}$ and the corresponding gauge mapping $k: M \rightarrow G L(V)$, we get the relation between $k$ and the uniformity mapping 
$P$ corresponding to the frame $\mathbf{p}$, namely, $\mathbf{p}_{i}(X)=P_{X}\left(\mathbf{e}_{i}\right)=\left(\mathbf{p}_{0}\right)_{i} k(X)=$ $P_{o, X}\left(\mathbf{e}_{i}\right) k(X)$, so that

$$
P_{X}=P_{o, X} \circ k(X) .
$$

These considerations are summarized in the following:

Proposition 1 Let $M$ be a simply connected parallelizable (i.e., admitting a global frame) manifold. With a choice of a reference configuration $\phi_{o}$ and a frame $\boldsymbol{e}_{i}$ in the archetype space $V$, there is a bijection between the following objects:

1. Global frames $\mathbf{p}$ in $M$ (global smooth sections of the frame bundle $F(M)$ );

2. Smooth uniformity mappings $P_{X}: V \rightarrow T_{X}(M)$;

3. Smooth mappings $\mathbf{k}: M \rightarrow G L(V), X \rightarrow \mathbf{k}(X)$ (elements of the gauge group $\left.G L(V)^{M}\right)$ such that for all $X \in M$

$$
\mathbf{p}(X)=\mathbf{p}_{o}(X) k(X)
$$

4. Nondegenerate smooth $(1,1)$-tensor fields $D_{J}^{I}(X)$ in $M$ such that

$$
\mathbf{D}(X) \mathbf{p}_{o}(X)=\mathbf{p}(X),
$$

or, in terms of uniformity mappings $P$ and $P_{o}$,

$$
\mathbf{D}(X)=P_{X} \circ P_{o}^{-1} .
$$

Remark 2 It is the bijection between the first two and the last types of geometrical objects (nondegenerate (1,1)-tensor fields) that will be primarily used in this paper.

\subsection{Uniform materials, II}

A uniformity mapping $P$ determines its own volume form by translating to the material the Euclidian volume element from the archetype: $v_{P}(X)=$ $P_{X}^{-1 *}\left(\mathbf{e}_{1} \wedge \mathbf{e}_{2} \wedge \mathbf{e}_{3}\right)$. Denote by $J_{P}(X)$ the factor relating two volume forms $v_{o}$ and $v_{P}$,

$$
v_{P}(X)=J_{P}(X) v_{0}(X)
$$

namely the Jacobian of the mapping $P^{-1}$. 
Comparing the definition of the factor $\lambda_{X}^{Y}$ in Eq. (4) with the definition of the factor $J_{P}(X)$, we get, for a uniform material, the following relation between these factors:

$$
\lambda_{X}^{Y}=\frac{J_{P}(X)}{J_{P}(Y)} .
$$

In terms of the volume factor $\mu_{P}$ uniformity condition (4) takes the form

$$
J_{P}^{-1}(Y) W(Y, F(Y))=J_{P}^{-1}(X) W\left(X, F(Y) \circ P_{Y} \circ P_{X}^{-1}\right) .
$$

Combining the deformation gradient $\mathbf{F}(X)$ and the uniformity mapping $P_{X}$, one gets the linear automorphism of the archetype space $A_{X}=\mathbf{F}(X) \circ P_{X} \in$ $G L(V)$. Comparing Eq. (4) with Eq. (8), we rewrite the condition (8) as follows:

$$
\begin{aligned}
& J_{P}^{-1}(X) W\left(X, \mathbf{F}(Y) \circ P_{Y} \circ P_{X}^{-1}\right) \\
& \quad=J_{P}^{-1}(Y) W(Y, \mathbf{F}(Y))=J_{P}^{-1}(Y) W\left(Y, \mathbf{F}(Y) \circ P(Y) \circ P(Y)^{-1}\right)
\end{aligned}
$$

for arbitrary points $X, Y \in M$ and an arbitrary value of the deformation gradient $\mathbf{F}(Y)$ at the point $Y$.

Define a function $\hat{W}$ of a point $X \in M$ and a linear mapping $A \in G L(V)$ by setting

$$
\hat{W}(X, A)=J_{P}^{-1}(X) W\left(X, A \circ P_{X}^{-1}\right) .
$$

In terms of the function $\hat{W}$, definition (8) of uniform material takes the very simple form

$$
\hat{W}(X, A)=\hat{W}(Y, A) .
$$

Thus, the uniformity condition (4) for the strain energy function $W$ is equivalent to the statement that the function $\hat{W}(X, A)), X \in M, A \in$ $G L(V)$ does not depend on the point $X \in M$. As a result, $\hat{W}(X, A)$ is a function on the linear group $G L(V)$ only. This result is the central point of the theory of (first-grade) uniform hyperelastic materials. It reduces the study of material properties of body $M$ and the evolution of those to the study of the uniformity mapping $P_{X}$ and the function $\hat{W}$ on the linear group $G L(V)$.

Additional physical requirements (e.g., material frame indifference, presence of a nontrivial material symmetry group, etc.) lead to additional restrictions on the form of the energy function $W$. For instance, material frame indifference requirement leads to the conclusion that $\hat{W}(A)$ is a function of invariants of 
matrix $A$. If a uniform material is isotropic, function $W(A)$ is left invariant with respect to the multiplication by elements of $S O(3)[11,13]$.

Returning to the the strain energy density function $W(X, F(X))$, we see that for a uniform material with the uniformity mapping $P$ the strain function $W$ takes the form $[10,12]$

$$
W(X, F(X))=J_{P}(X) \hat{W}(F(X) \circ P(X)) .
$$

\subsection{Material metric of a uniform structure}

As was already known to Cartan (see [19]), to a zero curvature linear connection $\omega$ (absolute parallelism) determined by a frame $\mathbf{p}$ (or by the corresponding uniformity map $P$ ) there corresponds the material metric $\mathbf{g}$ defined as the pullback of Euclidian metric $h$ by the mapping $P_{X}^{-1}$ :

$$
\mathbf{g}(X)=P_{X *}^{-1} \mathbf{h} .
$$

This definition is equivalent to declaring the frame $\mathbf{p}$ g-orthonormal at each point $X \in M$. In local coordinates $X^{I}$, the metric $\mathbf{g}$ has the form

$$
g_{I J}(X)=\left(P_{X}^{-1}\right)_{I}^{i}\left(P_{X}^{-1}\right)_{J}^{j} h_{i j}=\left(D(X)^{-1}\right)_{I}^{M}\left(D(X)^{-1}\right)_{J}^{N} g_{0 M N},
$$

the first expression being given in terms of the uniformity mapping $P$ while the second is in terms of the corresponding $(1,1)$-tensor field $\mathbf{D}$.

The curvature of the metric $\mathbf{g}$ is then defined by the torsion of the connection $\omega$ (see [16], eq. (34.19)).

\subsection{Examples}

Elastic strain tensor of a body in a configuration $\phi$ is defined by

$$
\mathbf{E}_{c}^{e l}=\frac{1}{2} \ln \left(\mathbf{g}_{0}^{-1} \mathbf{C}(\phi)\right) \approx \frac{1}{2}\left(g_{0}^{-1} C(\phi)-I\right),
$$

where the second expression is the linear approximation of the first one [15, 16]. Recall that the strain energy function of an isotropic material in linear elasticity has the form

$$
W(\phi)=\lambda\left[\operatorname{Tr}\left(\mathbf{E}^{e l}\right)\right]^{2}+\mu \operatorname{Tr}\left[\left(\mathbf{E}^{e l 2}\right)\right],
$$

where $\lambda, \mu$ are Lamé coefficients (see [15], Sec. 4.3). 
Using the same function $\hat{W}=\lambda[\operatorname{Tr}(A)]^{2}+\mu \operatorname{Tr}\left[A^{2}\right]$ on the linear group $G L(V)$ but a nontrivial uniformity mapping $P$, we come to the model of a quasi-isotropic material. Uniformity mapping $P$ defined the material metric $g$ as above. This allows us to redefine the elastic strain tensor using metric $g$ instead of the reference metric $g_{0}$ :

$$
\mathbf{E}^{e l}=\frac{1}{2} \ln \left(\mathbf{g}^{-1} \mathbf{C}(\phi)\right) \approx \frac{1}{2}\left(g_{0}^{-1} C(\phi)-I\right) .
$$

Strain energy of a quasi-isotropic material in linear elasticity is defied as follows:

$$
W_{P}(X, \mathbf{F}(X))=\mu_{P}(X)\left[\lambda\left(\operatorname{Tr}\left(\mathbf{E}^{e l}\right)\right)^{2}+\mu \operatorname{Tr}\left(\mathbf{E}^{e l 2}\right)\right] .
$$

It is easy to see that the strain energy is the quadratic function of the conventional elastic strain tensor $E_{c}^{e l}$ with the tensor of elastic moduli depending on the material point $X$.

Another example is provided by a quasi-Hookean material (see [15], p. 11), i.e., the uniform analog of the neo-Hookean material with

$$
W(\phi)=\alpha\left[\operatorname{Tr}\left(\mathbf{E}_{c}^{e l} 2\right)-3\right] .
$$

The quasi-Hookean material corresponding to a uniformity structure $P$ is defined by the same strain energy function but with the redefined strain tensor

$$
\mathbf{E}^{e l}=\frac{1}{2} \ln \left(\mathbf{g}^{-1} \mathbf{C}(\phi)\right),
$$

where material metric $g$ is used instead of the reference metric $g_{0}$ :

$$
\left.W_{P}(X, F(X))=\alpha\left(\operatorname{Tr}\left(\mathbf{E}^{e l 2}\right)-3\right)\right] .
$$

In the case of a homogeneous uniformity structure, the last expression reduces to the strain energy of standard neo-Hookean material.

\subsection{Evolution of the uniform structure}

Evolution of the properties of a uniform material is characterized by the timedependence of the uniformity mapping $P$ and that of the function $\hat{W}$. An appropriate characteristic of the evolution of the uniform structure $P$ is the material velocity $\mathbf{L}(X)$, which has been studied by different authors, see for instance $[5,12,20]$.

The material velocity of the uniformity structure $P$ is defined as the material point and time-dependent linear mapping,

$$
\mathbf{L}_{t}(X)=P_{X}^{-1} \circ \frac{\partial P_{X}}{\partial t}: V \rightarrow V .
$$


Under a loading, both the uniform structure $P$ and the deformation mapping $\phi$ are evolving. As a result, the couple $\left(P_{X}(t), \phi(t, X)\right)$ (or $(g(t, X), \phi(t, X))$ describes both the (total) deformation of a material and the evolution of its properties (elastic moduli, reference density, etc.). The rate of change of this couple is given by $\left(\mathbf{L}_{t}(X), \mathbf{V}(t, X)\right)$, where $\mathbf{V}(t, X)=\frac{\partial \phi}{\partial t}$ is the physical velocity.

\section{Elasto-plastic multiplicative decompositions of the deformation gradient}

At the end of 1950s, Bilby, Kröner ([2]) and later on Lee ([3]) proposed the following multiplicative decomposition of the deformation gradient (BKL decomposition),

$$
\mathbf{F}=\mathbf{F}^{e} \mathbf{F}^{p},
$$

as the product of two smooth $(1,1)$-tensor fields of elastic and plastic deformations, respectively. To provide a geometrical illustration of this decomposition, an intermediate configuration $C_{t}^{*}$ was introduced between the material body $M$ and the current configuration $C_{t}=\phi_{t}(M)$.

The decomposition $\mathbf{F}=\mathbf{F}^{e} \mathbf{F}^{p}$ is used to study the behavior exemplified by an elasto-plastic behavior of a material that undergoes deformation under a slowly applied load beyond the elastic range and then, after unloading, preserves some "permanent" strain (deformation). We refer to the works [4,21] for more examples and references concerning multiplicative decompositions of the deformation gradient $F$ and their applications.

\subsection{Relation between the BKL decomposition and the theory of uniform materials}

Recall that the deformation gradient $\mathbf{F}_{t}(X)$ of a configuration $\phi: M \rightarrow E^{3}$ is the two-point (1,1)-tensor field in $M$ defined by the linear isomorphism of the tangent spaces $\phi_{*}: T(M) \rightarrow T\left(\phi_{t}(M)\right)$ at $X \in M$. Here $C_{t}=\phi_{t}(M)$ is the configuration of the body at the time $t$.

The decomposition (16) can be hardly interpreted other than as the composition of tangent bundle mappings over some mappings of corresponding base manifolds:

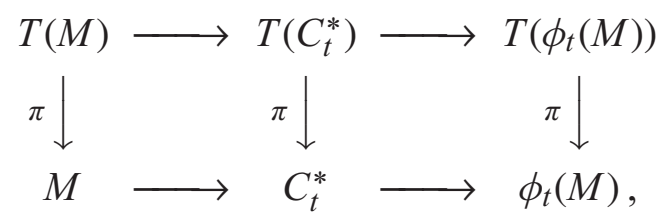


since the tensor fields $\mathbf{F}^{e}$ and $\mathbf{F}^{p}$ should be strictly anchored at some manifolds (domain and target of each). Moreover, the first mapping $\mathbf{F}^{p}$ should define a mapping from the tangent space $T_{X}(M)$ at a point $X \in M$ to the tangent space at some point $Y_{X}$ of intermediate configuration $C_{t}^{*}$. The correspondence $X \rightarrow Y_{X}$ should be one-to-one, otherwise the composition of mappings (16) cannot be an isomorphism of the tangent bundles. Therefore, there exists a unique one-to-one mapping $\phi_{1}: M \rightarrow C_{t}^{*}$ underlying the tangent bundle mapping $\mathbf{F}^{p}$. Mapping $\phi_{1}$ can be assumed to be differentiable.

In the same way, $\mathbf{F}^{e}$ can be viewed as a mapping of tangent bundles $T\left(C_{t}^{*}\right) \rightarrow$ $T\left(C_{t}\right)$ over the differentiable mapping $\phi_{2}: C_{t}^{*} \rightarrow T\left(C_{t}\right)$ of basis manifolds.

We obviously have $\phi=\phi_{2} \circ \phi_{1}$. Therefore, $\phi_{2}$ is onto. Restricting, if necessary, the intermediate configuration manifold, one may assume, without losing generality, that $\phi_{1}$ is onto and $\phi_{2}$ is one to one. Thus, both $\phi_{1}$ and $\phi_{2}$ can be considered as diffeomorphisms.

Remark 3 Defining the decomposition (16), some authors presume that the mappings $\mathbf{F}^{e}$ and $\mathbf{F}^{p}$ are nonsmooth or even noncontinuous, reflecting microdefect densities in the manifold $M$. Translating this into the language of tensor fields and using the derivatives of these tensor fields, one should however assume some smoothness. Usually it is done by considering these tensor fields as smooth averaged characteristics of the structural state of the material.

Remark 4 Mapping $\phi_{1}$ presents the intermediate configuration introduced in 1960 by a variety of researchers; see [3,22,23]. It was used for the construction of plastic deformation gradient $F^{p}$ and the elasto-plastic decompositions of total deformation gradient $F$, but as far as we know, was not considered previously as an independent dynamical variable.

Now we are ready to take the next step. Consider the tangent mapping $\phi_{1 t^{*}}$ : $T(M) \rightarrow T\left(C_{t}^{*}\right)$ and compare it with the mapping $\mathbf{F}^{p}(t, X): T(M) \rightarrow T\left(C_{t}^{*}\right)$. Since mapping $\phi_{1 t *}$ is linear isomorphism at each point $X \in M$, one can write, for all tangent vectors $\xi \in T_{X}(M)$,

$$
\mathbf{F}^{p}(t ;(X, \xi))=\phi_{1 t * X} \circ \mathbf{D}_{t}(X) \cdot \xi,
$$

where $\mathbf{D}_{t}(X)$ is uniquely defined smooth $(1,1)$-tensor field in $M$.

In exactly the same way, one can present

$$
\mathbf{F}^{e}(t, Y, \eta)=\phi_{2 t * Y} \circ \mathbf{F}^{e *}(t, Y) \cdot \xi
$$

for the uniquely defined smooth $(1,1)$-tensor field $\mathbf{F}^{e *}(t, Y)$ in $C_{t}^{*}$. 
If we pull back the (spatial index of) tensor field $\mathbf{F}^{e *}(t, Y)$ from $C_{t}^{*}$ onto $M$ by the differential $\phi_{1 \text { t* }}$ of the point mapping $\phi_{1}$, we get another $(1,1)$-tensor $\mathbf{D}^{e}$ on $M$.

Since $\phi(t, X)=\phi_{t 2} \circ \phi_{t 1}$ for all $t$ and, therefore, $\phi(t, X)_{* X}=\phi_{t 2 * \phi_{t 1}(\mathbf{X})} \circ$ $\phi_{t 1 * X}$, combining this with the decomposition $(\star)$ we get

$$
\begin{aligned}
\phi_{*} & =\mathbf{F}^{e} \circ \mathbf{F}^{p}=\left(\phi_{2 *} \circ \mathbf{D}^{e}\right) \circ\left(\phi_{1 *} \circ \mathbf{D}\right)=\left(\phi_{2 *} \circ \phi_{1 *}\right) \circ\left(\phi_{1 *}^{-1} \circ \mathbf{D}^{e} \circ \phi_{1 *}\right) \circ \mathbf{D} \\
& =\left(\phi_{2} \circ \phi_{1}\right)_{*} \circ\left(\mathbf{D}^{e} \circ \mathbf{D}\right)=\phi_{*} \circ\left(\mathbf{D}^{e} \circ \mathbf{D}\right),
\end{aligned}
$$

so that

$$
\mathbf{D}^{e}(t, X) \cdot \mathbf{D}(t, X)=i d_{T(M)} .
$$

As a result, being transferred to the material manifold $M$, the (1,1)-tensor fields connecting integrable mappings $\phi_{i *}(i=1,2)$ to the tangent bundle mappings $\mathbf{F}^{p}$ and $\mathbf{F}^{e}$ are inverse to one other. This is hardly a surprise since in the physical literature only one of these tensors was considered an independent dynamical variable; see $[4,24]$.

In the same way, $\phi_{2}=\phi \circ \phi_{1}^{-1}$ would also be redundant. As a result, the only independent dynamical variables in this scheme are diffeomorphic embeddings $\phi, \phi_{1}$ and the material $(1,1)$-tensor field $\mathbf{D}$.

Remark 5 One can of course choose another triple of variables as independent dynamical quantities, for instance one may use $\left(\phi_{1}, \phi_{2}, \mathbf{D}\right)$ if it is preferable to deal with the elastic deformation $\phi_{2}$ explicitly.

Remark 6 We consider here only the decomposition $\mathbf{F}=\mathbf{F}^{e} \mathbf{F}^{p}$, but the same arguments would produce a geometrical representation of the reverse $\mathbf{F}=$ $\mathbf{F}^{p} \mathbf{F}^{e}$ decomposition as well.

Remark 7 Notice that the choice of an intermediate configuration $\left(C_{t}^{*}, \phi_{1 t}\right)$ participating in the decomposition $(\star)$ is far from being unique. In particular, let us show that we may formally choose the image $C_{t}^{*}=\phi_{o}(M)$ as the intermediate configuration with $\phi_{1 t}=\phi_{o}$ being time independent. To do this, denote by $\psi: C_{t}^{*} \rightarrow C_{o}$ the diffeomorphism $\psi=\phi_{o} \circ \phi_{1}^{-1}$. Transfer the tensor $\mathbf{F}^{e}$ to $C_{o}$ as follows: $\mathbf{F}^{e} 0=\psi_{*}\left(\mathbf{F}^{e *}\right)$, where we are using the diffeomorphism $\psi$ together with its inverse to push forward the $(1,1)$-tensor $\mathbf{F}^{\mathbf{e} *}$. Thus we get the mapping of tangent bundles

$$
\hat{\mathbf{F}} \mathbf{F}^{e}=\chi_{t *} \circ \mathbf{F}^{e o}=\chi_{t *} \circ \psi_{t *} \circ \mathbf{F}^{e *} \circ \psi_{t *}^{-1} .
$$


Define also the diffeomorphism $\chi_{t}: C_{o} \rightarrow C_{t}$ as $\chi_{t}=\phi \circ \phi_{o}^{-1}$. and define the mapping of the tangent bundles by setting

$$
\hat{\mathbf{F}}^{p}=\phi_{o *} \circ \mathbf{F}^{p M} \text {. }
$$

Then we have $\phi=\chi \circ \phi_{o}$, and, as is easy to check, $\hat{\mathbf{F}}^{e} \circ \hat{\mathbf{F}}^{p}=\phi_{*}$ as required.

As a result, we get a simplified scheme of the elastic-plastic $\mathbf{F}^{e} \mathbf{F}^{p}$ decomposition of the deformation gradient $F=\phi_{*}$ of a uniform material. Notice that the integrable part $\phi_{1} t$ of the plastic deformation gradient $\mathbf{F}^{p}$ is lost in this simplified scheme. That is why it is preferable to work with the previous scheme where the intermediate configuration is different from the image of the reference embedding $\phi_{o}$.

Remark 8 Notice that the couple $\left(\mathbf{D}, \phi_{1}\right)$ represents another model of evolution of the material of the same type with the same uniformity structure. This model of pure inelastic evolution is related to the model $\left(\phi, \phi_{1}, D\right)$ by the elastic deformation $\phi_{2}$.

If we start with a time-dependent uniformity mapping $P_{t}$ and two configurations $\phi, \phi_{1}: M \rightarrow R^{3}$, then one can (reversing the arguments above) construct the "elastic deformation" $\phi_{2}=\phi \circ \phi_{1}^{-1}$ and the mappings of tangent bundles $\mathbf{F}^{p}: T(M) \rightarrow T\left(C^{*}=\operatorname{Im}\left(\phi_{1, t}\right), \mathbf{F}^{e}: T\left(C_{t}^{*}=\operatorname{Im}\left(\phi_{1, t}\right) \rightarrow T\left(C_{t}=\operatorname{Im}\left(\phi_{t}\right)\right)\right.\right.$, such that the construction above returns us to the triple $\left(P_{t}, \phi, \phi_{1}\right)$.

Finally, there is freedom in the choice of the decomposition $\mathbf{F}^{p}=\phi_{1 *} \circ \mathbf{D}$ given by an arbitrary diffeomorphism $\psi \in \operatorname{Diff}(M)$ :

$$
\mathbf{F}^{p}=\phi_{1 *} \circ \mathbf{D}=\left(\phi_{1} \circ \psi^{-1}\right)_{*} \circ\left(\psi_{*} \circ \mathbf{D}\right) .
$$

Thus, we can introduce the following equivalence relation between the pairs $\left(\phi_{1}, \mathbf{D}\right)$ of the (time-dependent) mappings $\phi_{1}: M \rightarrow R^{n}$ and nondegenerate $(1,1)$-tensor fields $\mathbf{D}$ in $M$. We say that two pairs $\left(\phi_{1}, \mathbf{D}\right),\left(\chi_{1}, K\right)$ are equivalent if there is a diffeomorphism $\psi \in \operatorname{Diff}(M)$ such that

$$
\chi_{1}=\phi_{1} \circ \psi^{-1}, K=\psi_{*} \circ \mathbf{D} .
$$

Collecting the considerations presented in this section, we come to the following conclusions:

1. The BKL decomposition $F=F^{p} F^{e}$ of the deformation gradient $F=\phi_{*}$ of a (total) configuration $\phi_{t}: M \rightarrow E^{3}$ presupposes the existence of (intermediate) inelastic configuration $\phi_{1 t}: M \rightarrow E^{3}$ and of the nondegenerate $(1,1)$-tensor field $D_{t}$ in the material space $M$ such that 


$$
\begin{aligned}
& \mathbf{F}^{p}=\phi_{1 *} \circ \mathbf{D}, \\
& \mathbf{F}^{e}=\phi_{*} \circ \mathbf{D}^{-1} \circ \phi_{1 *}^{-1} .
\end{aligned}
$$

2. Configuration $\phi_{1}$ is the mapping $\phi_{1 t}: M \rightarrow E^{3}$ defining the integrable part of inelastic (plastic!) deformation gradient $\mathbf{F}^{p}$.

3. (1,1)-tensor $\mathbf{D}$ is equal to

$$
\mathbf{D}_{t}=\phi_{1 *}^{-1} \circ \mathbf{F}^{p}(t, X) .
$$

4. Vice versa, to any triple $\left(\phi_{t}, \mathbf{F}_{t}^{p}, \mathbf{F}_{t}^{e}\right)$ consisting of two configurations and a nondegenerate $(1,1)$-tensor field $D_{t}$ in $M$ there corresponds the multiplicative decomposition

$$
\phi_{*}=F=\mathbf{F}^{e} \circ \mathbf{F}^{p}
$$

of the deformation gradient $F$ of the total deformation history $\phi_{t}$.

5. Tensor field $D_{t}$ defines the time-dependent uniform structure $P_{t}=D_{t} \circ P_{0}$ (see Proposition 1) in the material body $M$.

6. Uniform structure $D$ determines the time-dependent Riemannian metric $\mathbf{g}_{t}$ in the material manifold $M$ (see Section 2.6) by the formula:

$$
g_{t I J}=h_{i j} D_{I}^{i} D_{J}^{j}
$$

Remark 9 Notice that the decomposition $\mathbf{F}=\phi_{*}=\mathbf{F}^{e} \circ \mathbf{F}^{p}$ determines the tensor field $\mathbf{D}$ and the plastic integrable deformation $\phi_{1}$ up to an action of a diffeomorphism $\chi$.

On the other hand, there are arguments showing that the inelastic configuration $\phi_{1}$ is defined uniquely by the history of deformation. If total deformation $\phi_{0} \rightarrow \phi_{t}$ is subject to certain conditions of loading, heating, etc., the unloading or turning of the heat at some moment $t_{1}$ produces certain configuration $\phi_{1}: M \rightarrow E^{3}$. Often the unloading happens fast and is not accompanied by an essential change in the material structure. See [25] where relaxation of a material to the intermediate configuration $\phi_{1}$ during unloading is discussed. As a result, we may associate with the moment $t_{1}$ the final configuration $\phi_{1} t_{1}$ taken by the body $M$ after unloading. As a result, under physically reasonable assumptions on the evolution process, the intermediate configuration $\phi_{1}$, and therefore the tensor field $\mathbf{D}$, are determined uniquely (up to a composition of $\phi_{1}$ with the Euclidean motion of the physical space $\left.\mathbf{E}^{3}\right)$. 
Using the terminology of Maugin and Muschik [26], the configuration $\phi_{1}$ is "observable but not controllable" (or, citing the same work, "partly controllable" through the loading conditions).

In this analysis of the kinematics of the BKL decomposition, we identified three variables: the uniform structure $P_{t}$, the integrable part of the "plastic deformation gradient" $\phi_{1}$ (intermediate configuration), and the total deformation $\phi$, which we will consider as physically independent. Thus, the full dynamical/thermodynamical picture with these kinematics should include all three components. Below, discussing the dynamical structure of the presented model, we will be using material metric $g$ instead of the tensor field $D$ as the material dynamical variable. Metric $g$ contains the essential information about the uniformity structure $P_{t}$ and is more convenient to use when describing the evolution of material properties than the tensor $D$ or uniformity mapping $P$; see [11], Ch. 11.

\section{Strain tensors: elastic, inelastic, and metric}

\subsection{Strain tensors}

Nonlinear elasticity theory has, as its geometrical keystone, the question of the comparison of two metrics: the reference metric $\mathbf{g}_{o}$ and the Cauchy metric $\mathbf{C}\left(\phi_{t}\right)$ of a configuration $\phi_{t},[15]$.

As Theorem 1 shows, the multiplicative decomposition leads, in its geometrical form, to the presence of four metrics in the material manifold $M$ : the reference metric $\mathbf{g}_{o}$, the material metric $\mathbf{g}$ generated by the $(1,1)$-tensor field $\mathbf{D}=\mathbf{F}^{p M}$, the Cauchy metric of the integrable part of plastic deformation $\mathbf{C}\left(\phi_{1}\right)$, and, finally, the Cauchy metric of the total deformation $\mathbf{C}(\phi)$. It seems natural to define appropriate strain tensors as measures of comparison between pairs of these metrics and use these tensors for description of different processes developing in the material.

We will introduce six strain tensors as suited to describe the state of our solid and characterize specific processes undergoing in the body.

\section{Elastic strain tensor:}

$$
\begin{aligned}
\mathbf{E}^{e l} & =\frac{1}{2} \ln \left[\mathbf{C}\left(\phi_{1}\right)^{-1} \mathbf{C}(\phi)\right] \\
& \approx \frac{1}{2} \mathbf{C}\left(\phi_{1}\right)^{-1}\left[\mathbf{C}(\phi)-\mathbf{C}\left(\phi_{1}\right)\right] \approx \frac{1}{2} \mathbf{g}^{-1}\left[\mathbf{C}(\phi)-\mathbf{C}\left(\phi_{1}\right)\right] .
\end{aligned}
$$

Elastic strain tensor measures the elastic part of the deformation at each instant of time and vanishes under unloading. Tensor $E^{e l}$ and its linearized 
variant are $C\left(\phi_{1}\right)$-symmetrical (i.e., $E_{I J}^{e l}=C\left(\phi_{1}\right)_{I K} E_{J}^{e l} K$ is symmetrical $(0,2)$-tensor).

\section{Inelastic strain tensor:}

$$
\mathbf{E}^{i n}=\frac{1}{2} \ln \left[\mathbf{g}^{-1} \mathbf{C}\left(\phi_{1}\right)\right] \approx \frac{1}{2} \mathbf{g}^{-1}[\mathbf{C}(\phi)-\mathbf{g}] \approx \frac{1}{2} \mathbf{g}_{o}^{-1}[\mathbf{C}(\phi)-\mathbf{g}] .
$$

Inelastic (plastic) strain tensor measures the plastic but still Euclidean deformation of the body, i.e., permanent after unloading but not leading to any residual stresses in the material. Tensor $E^{i n}$ and its linearized version $E_{\text {lin }}^{\text {in }}$ are $g$-symmetrical, i.e., $(0,2)$-tensors $g_{I K} E_{J}^{\text {in } K}, g_{I K} E_{\text {lin } J}^{\text {in } K}$ are symmetrical.

\section{Material strain tensor:}

$$
\mathbf{E}^{m}=\frac{1}{2} \ln \left(\mathbf{g}_{o}^{-1} \mathbf{g}\right) \approx \frac{1}{2} \mathbf{g}_{o}^{-1}\left(\mathbf{g}-\mathbf{g}_{o}\right) .
$$

Material strain tensor measures the pure metrical evolution of the material, not leading to any deformation (material points displacement). Tensor $E^{m}$ and its linearized version $E_{\text {lin }}^{m}$ are $g_{0}$-symmetrical.

\section{Euclidian strain tensor:}

$$
\mathbf{E}^{\text {eucl }}:=\frac{1}{2} \ln \left[\mathbf{g}^{-1} \mathbf{C}(\phi)\right] \approx \frac{1}{2} \mathbf{g}^{-1}[\mathbf{C}(\phi)-\mathbf{g}] .
$$

Euclidian strain tensor measures the integrable part of the total deformation.

\section{Total strain tensor:}

$$
\mathbf{E}^{t o t}=\frac{1}{2} \ln \left(\mathbf{g}_{o}^{-1} \mathbf{C}(\phi)\right) \approx \frac{1}{2} \mathbf{g}_{o}^{-1}\left(\mathbf{C}(\phi)-\mathbf{g}_{o}\right) .
$$

Total strain tensor measures the decline of the Cauchy metric of total deformation $\phi$ from the reference (euclidian) metric $\mathbf{g}_{o}$. It is observable. Tensor $E^{\text {tot }}$ and its linearized version $E_{\text {lin }}^{\text {tot }}$ are $g_{0}$-symmetrical.

\section{Total inelastic strain tensor:}

$$
\mathbf{E}^{t i n}=\frac{1}{2} \ln \left(\mathbf{g}_{o}^{-1} \mathbf{C}\left(\phi_{1}\right)\right) \approx \frac{1}{2} \mathbf{g}_{o}^{-1}\left(\mathbf{C}\left(\phi_{1}\right)-\mathbf{g}_{o}\right) .
$$

Total inelastic strain tensor measures the decline of the Cauchy metric of inelastic deformation $\phi_{1}$ from the reference (euclidian) metric $\mathbf{g}_{o}$. It is observable (after unload).

In each case we provide the linear approximation form(s) of the strain tensors suited for small deviation of the former metric from the latter one. 
Remark 10 In some simple cases, say when the $(1,1)$-tensors $\mathbf{g}^{-1} \mathbf{C}\left(\phi_{1}\right)$ and $\mathbf{C}\left(\phi_{1}\right)^{-1} \mathbf{C}(\phi)$ commute, from the relation $\mathbf{g}^{-1} \mathbf{C}(\phi)=\mathbf{g}^{-1} \mathbf{C}\left(\phi_{1}\right) \cdot \mathbf{C}\left(\phi_{1}\right)^{-1}$ $\mathbf{C}(\phi)$ we conclude that the linearized $\mathbf{E u c l i d e a n}$ strain tensor $\mathbf{E}^{\text {eucl }}$ splits as follows:

$$
\begin{aligned}
\mathbf{E}^{\text {eucl }} & \approx \frac{1}{2} \mathbf{g}^{-1}[\mathbf{C}(\phi)-\mathbf{g}]=\frac{1}{2} \mathbf{g}^{-1}\left[\mathbf{C}(\phi)-\mathbf{C}\left(\phi_{1}\right)\right]+\frac{1}{2} \mathbf{g}^{-1}\left[\mathbf{C}\left(\phi_{1}\right)-\mathbf{g}\right] \\
& \approx \mathbf{g}^{-1} \mathbf{C}\left(\phi_{1}\right) \mathbf{E}^{e l}+\mathbf{E}^{m} \approx \mathbf{E}^{e l}+\mathbf{E}^{i n} .
\end{aligned}
$$

If all three (1,1)-tensors $\mathbf{g}_{o}^{-1} \mathbf{g}, \mathbf{g}^{-1} \mathbf{C}\left(\phi_{1}\right)$, and $\mathbf{C}\left(\phi_{1}\right)^{-1} \mathbf{C}(\phi)$ commute, we have an additive decomposition of the linearized total strain tensor $\mathbf{E}^{\text {tot }}$ :

$$
\begin{aligned}
\mathbf{E}^{t o t} & \approx \frac{1}{2} \mathbf{g}_{0}^{-1}\left[\mathbf{C}(\phi)-\mathbf{g}_{o}\right]=\frac{1}{2} \mathbf{g}_{0}^{-1}\left[\mathbf{C}(\phi)-\mathbf{C}\left(\phi_{1}\right)+\mathbf{C}\left(\phi_{1}\right)-\mathbf{g}+\mathbf{g}-\mathbf{g}_{o}\right] \\
& =\frac{1}{2} \mathbf{g}_{0}^{-1} \mathbf{C}\left(\phi_{1}\right) \mathbf{C}\left(\phi_{1}\right)^{-1}\left[\mathbf{C}(\phi)-\mathbf{C}\left(\phi_{1}\right)\right]+\frac{1}{2} \mathbf{g}_{0}^{-1} \mathbf{g g}^{-1}\left[\mathbf{C}\left(\phi_{1}\right)-\mathbf{g}\right]+\frac{1}{2} \mathbf{g}_{0}^{-1}\left(\mathbf{g}-\mathbf{g}_{o}\right) \\
& =\mathbf{E}^{m}+\mathbf{g}_{0}^{-1} \mathbf{g} \mathbf{E}^{i n}+\mathbf{g}_{0}^{-1} \mathbf{C}\left(\phi_{1}\right) \mathbf{E}^{e l} \approx \mathbf{E}^{m}+\mathbf{E}^{i n}+\mathbf{E}^{e l} .
\end{aligned}
$$

\subsection{Choice of the dynamical variables}

In order to determine which combinations of dynamical variables $\left(\phi, \phi_{1}, D\right)$ and their derivatives might enter the internal or free energy, dissipative potential, entropy, and other dynamical and thermodynamical quantities, we have to take into account requirements of invariance or covariance of these quantities with respect to the appropriate material and spatial transformations. For instance, the frame indifference requirement $[27,28]$ leads to the conclusion that the deformation gradient $F(X)$ of the total deformation $\phi$ enters these quantities only in combinations $C\left(\phi_{1}\right)^{-1} C(\phi), g_{0}^{-1} C(\phi)$, or $g^{-1} C(\phi)$.

Material metric $\mathbf{g}$ and the Cauchy-Green tensor $\mathbf{C}\left(\phi_{1}\right)$ depend on the choice of the plastic decomposition (19). Thus, it is important to determine which tensors or combinations of tensors constructed from the dynamical fields $\left(\phi, \phi_{1}, \mathbf{D}\right)$ are independent of the choice of the plastic decomposition (19).

We consider several such combinations.

1. The total strain tensor

$$
\mathbf{E}^{t o t}=\frac{1}{2} \ln \left(\mathbf{g}_{o}^{-1} \phi^{*} \mathbf{h}\right) \approx \frac{1}{2} \mathbf{g}_{o}^{-1}\left[\mathbf{C}(\phi)-\mathbf{g}_{o}\right]
$$

is independent of the choice of a plastic decomposition (19). Since it is a $(1,1)$-tensor, its invariants are independent of the choice of a decomposition (19) and, moreover, one can combine it with other similar tensors to produce new invariant combinations. 
2. The plastic deformation gradient $\mathbf{F}^{p}=\phi_{1 * \circ} \mathrm{D}$ does not depend on the choice of a decomposition (19) but it is convenient to transform it into a material tensor. For instance, one can use the following variant of the Cauchy-Green tensor:

$$
\mathbf{F}^{p *} \mathbf{h} \equiv \mathbf{D}^{*}\left(\phi_{1}^{*} \mathbf{h}\right)=\mathbf{D}^{*} \mathbf{C}\left(\phi_{1}\right) .
$$

Lifting one index in this tensor by means of the reference metric $\mathbf{g}_{0}$ we get the material (1,1)-tensor $\mathbf{g}_{o}^{-1 *}{ }^{*} \mathbf{F}^{p *} \mathbf{h}$. This tensor carries information on both integrable and nonintegrable parts of the plastic deformations.

3. The material metric $\mathbf{g}=P^{-1 *} \mathbf{h}$ can be written in a number of different ways. For instance, by using the reference metric $\mathbf{g}_{o}=P_{o}^{-1 *} \mathbf{h}$, we can get

$\mathbf{g}=P^{-1 *} \mathbf{h}=P^{-1 *}\left(P_{o}^{*} \mathbf{g}_{o}\right)=\left(P_{o} \circ P^{-1}\right)^{*} \mathbf{g}_{o}=\left(P \circ P_{o}^{-1}\right)^{-1 *} \mathbf{g}_{o}=\mathbf{D}^{-1 *} \mathbf{g}_{o}$.

In local coordinates we have

$$
g_{A B}=\left(D^{-1}\right)_{A}^{M}\left(D^{-1}\right)_{B}^{N} g_{o} M N, g^{-1 A B}=D_{M}^{A} D_{N}^{B} g_{0}^{M N} .
$$

Under the change of decomposition (19), the material metric $\mathbf{g}$ is transformed by a diffeomorphism $\psi$ into a new metric $\mathbf{g}^{\psi}$ as follows:

$$
\mathbf{g}^{\psi}=\left(\psi_{*} \circ \mathbf{D}\right)^{-1 *} \mathbf{g}_{0}=\left(\mathbf{D}^{-1} \circ \psi_{*}\right)^{*} \mathbf{g}_{0}=\psi^{-1 *} \circ \mathbf{D}^{-1 *} \mathbf{g}_{0}=\psi^{*} \mathbf{g} .
$$

In coordinates,

$$
\left(g^{\psi}\right)_{A B}=\left(\psi^{-1}\right)_{A}^{M}\left(\psi^{-1}\right)_{B}^{N} g_{M N},\left(g^{\psi-1}\right)^{C D}=\psi_{M}^{C} \psi_{N}^{D} g^{-1 M N} .
$$

Therefore, the curvature tensor $R_{J K L}^{I}$ (as well as the corresponding Ricci tensor $R_{I J}(\mathbf{g})$ ) is transformed tensorially by $\psi^{*}$ or $\psi_{*}$. Thus, invariants of these curvature tensors (in particular the scalar curvature $R(\mathbf{g})$ ), do not depend on a choice of plastic decomposition (19).

4. At the same time the Cauchy metric of the inelastic deformation $\mathbf{C}\left(\phi_{1}\right)$ is transformed by $\psi^{-1}$ tensorially as well:

$$
\begin{aligned}
C\left(\phi_{1} \circ \psi^{-1}\right)_{A B} & =\left(\psi^{-1 *} \circ \phi_{1}^{*} h\right)_{A B}=\left[\psi^{-1 *} C\left(\phi_{1}\right)\right]_{A B} \\
& =\left(\psi^{-1}\right)_{A}^{M}\left(\psi^{-1}\right)_{B}^{N} C\left(\phi_{1}\right)_{M N} .
\end{aligned}
$$

Combining the material metric and the Cauchy tensor of the inelastic deformation $\phi_{1}$, we finally get the inelastic strain tensor $\mathbf{E}^{\text {in }}$. For a different decomposition (19), we have

$$
\begin{aligned}
& \left(g^{\psi}-1\right)^{A C} C\left(\phi_{1} \circ \psi^{-1}\right)_{C B}=\psi_{M}^{A} \psi_{N}^{C} g^{-1 M N}\left(\psi^{-1}\right)_{C}^{K}\left(\psi^{-1}\right)_{B}^{L} C\left(\phi_{1}\right)_{K L} \\
& \quad=\psi_{M}^{A} g^{-1 M N} \delta_{N}^{K}\left(\psi^{-1}\right)_{B}^{L} C\left(\phi_{1}\right)_{K L}=\psi_{M}^{A}\left(\psi^{-1}\right)_{B}^{L} g^{-1 M K} C\left(\phi_{1}\right)_{K L} \\
& \quad=\psi_{M}^{A}\left(\psi^{-1}\right)_{B}^{L}\left(g^{-1} C\left(\phi_{1}\right)\right)_{L}^{M} .
\end{aligned}
$$


Thus, $\mathbf{E}^{\text {in }}$ transforms tensorially under a change of plastic decomposition (19) and its invariants are independent of this decomposition.

The presence of the two quantities $\mathbf{F}^{p}$ and $\mathbf{E}^{i n}$, whose invariants are independent of the choice of plastic decomposition, makes it important to compare these two quantities. We have

$$
\begin{aligned}
& \left.\mathbf{g}_{o}^{-1}\left(\mathbf{F}^{p *}\right) \mathbf{h}\right)=\mathbf{g}_{o}^{-1}\left(\mathbf{D}^{*} \mathbf{C}\left(\phi_{1}\right)\right)=g_{o}^{-A C} D_{C}^{M} D_{B}^{N} C\left(\phi_{1}\right)_{M N} \\
& \quad=D_{L}^{-1 A}\left(g_{o}^{-1 K C} D_{K}^{L} D_{C}^{M}\right) C\left(\phi_{1}\right)_{M N} D_{B}^{N}=D_{L}^{-1 A}\left(g^{-1 M L}\right) C\left(\phi_{1}\right)_{M N} D_{B}^{N} \\
& \quad=D_{L}^{-1 A}\left(g^{-1} C\left(\phi_{1}\right)\right)_{N}^{L} D_{B}^{N}=D_{L}^{-1 A}\left(\exp \left(2 E^{i n}\right)\right)_{N}^{L} D_{B}^{N},
\end{aligned}
$$

i.e.,

$$
\mathbf{g}_{o}^{-1}\left(\mathbf{F}^{p *} \mathbf{h}\right)=D_{L}^{-1 A}\left[\exp \left(2 E^{i n}\right)\right]_{N}^{L} D_{B}^{N},
$$

and therefore invariants of the tensor $\mathbf{g}_{o}^{-1}\left(\mathbf{F}^{p *} \mathbf{h}\right)$ (i.e., tensorial characteristics of the plastic gradient deformation $\mathbf{F}^{p}$ ) contain the same information as the invariants of $\mathbf{E}^{\text {in }}$.

Thus, in the restricted case, it seems natural to choose an internal energy $u$ as a function of the invariants of the two strain tensors $\mathbf{E}^{\text {tot }}$ and $\mathbf{E}^{\text {in }}$, of the invariants of the curvature tensor $R_{j k l}^{i}(\mathbf{g})$ (of the Ricci Tensor $\operatorname{Ric}(\mathbf{g})_{J}^{I}$ in the 3-dim case, see [17], Ch. 6, Sec. 5), temperature, and its $\mathbf{g}_{o}$-gradient: $u=u\left(\left[\mathbf{E}^{t o t}(\phi), \mathbf{E}^{i n}\left(\phi_{1}, \mathbf{g}\right), \operatorname{Ric}(\mathbf{g}), \theta, \nabla^{\mathbf{g}_{o}} \theta\right]\right)$.

If we adopt the assumptions of Remark 5 (i.e., removing the restriction to use only tensors that do not depend on a choice of plastic decomposition (19)), we may consider all three strain tensors $\mathbf{E}^{t o t}, \mathbf{E}^{i n}, \mathbf{E}^{m}$ (one can replace $\mathbf{E}^{t o t}$ in this list by $\mathbf{E}^{e l}$ if it is preferable) as independent dynamical variables and, together with the Ricci tensor of the material metric $\mathbf{g}$, include them as arguments in the internal energy:

$$
u=u\left[\mathbf{E}^{e l}, \mathbf{E}^{i n}, \mathbf{E}^{m}, \operatorname{Ric}(\mathbf{g}), \theta, \nabla^{\mathbf{g}_{o}} \theta\right] .
$$

In this approach, the effects of different types of processes are directly separated.

\subsection{Additional strain decompositions}

Between the strain tensors introduced above, the conventional strain tensors, and both deformation gradients $F^{e}, F^{p}$ of the multiplicative decomposition, there exist different relations that may be in some partial cases more convenient than those presented above. Below are two examples of such relations, the first being valid in the linear case, the second in a nonlinear situation. 


\section{Linear case:}

Since $F=\phi_{*}=\mathbf{F}^{e} \circ \mathbf{F}^{p}$, we have

$$
\mathbf{g}_{o}^{-1} \phi^{*} \mathbf{h}=\mathbf{g}_{o}^{-1} \mathbf{F}^{p *}\left(\mathbf{F}^{e *} \mathbf{h}-\mathbf{h}+\mathbf{h}\right)=\mathbf{g}_{o}^{-1} \mathbf{F}^{p *} \mathbf{h}+\mathbf{g}_{o}^{-1} \mathbf{F}^{p *}\left(\mathbf{F}^{e *} \mathbf{h}-\mathbf{h}\right)
$$

and therefore

$$
\begin{aligned}
\mathbf{E}_{\text {lin }}^{\text {tot }} & =\frac{1}{2}\left(\mathbf{g}_{o}^{-1} \phi^{*} \mathbf{h}-\boldsymbol{\delta}\right)=\frac{1}{2}\left(\mathbf{g}_{o}^{-1} \mathbf{F}^{p *} \mathbf{h}-\boldsymbol{\delta}\right)+\mathbf{g}_{o}^{-1} \mathbf{F}^{p *} \frac{1}{2}\left(\mathbf{F}^{e *} \mathbf{h}-\mathbf{h}\right) \\
& \approx \mathbf{D}^{-1} \mathbf{E}_{\text {lin }}^{\text {in }} \mathbf{D}+\mathbf{g}_{o}^{-1} \mathbf{F}^{p *} \mathbf{E}_{\text {lin }}^{e l},
\end{aligned}
$$

where we defined

$$
\mathbf{E}_{\text {lin }}^{e l}=\frac{1}{2}\left(\mathbf{F}^{e *} \mathbf{h}-\mathbf{h}\right) .
$$

This decomposition can be compared with those in Section 4.1.

2. Nonlinear case:

$$
\begin{aligned}
\mathbf{E}^{t o t}= & \frac{1}{2} \ln \left(\mathbf{g}_{o}^{-1} \phi^{*} \mathbf{h}\right)=\frac{1}{2} \ln \left(\mathbf{g}_{o}^{-1}\left(\mathbf{F}^{p *} \mathbf{h}\right) \mathbf{h}^{-1}\left(\mathbf{F}^{e *} \mathbf{h}\right)\right) \\
= & \frac{1}{2} \ln \left(\mathbf{g}_{o}^{-1}\left(\mathbf{F}^{p *} \mathbf{h}\right) \cdot \exp \left(2 \mathbf{E}_{\text {old }}^{e l}\right)\right) \\
= & \frac{1}{2} \ln \left(\mathbf{g}_{o}^{-1}\left(\mathbf{F}^{p *} \mathbf{h}\right) \cdot\left(\mathbf{F}^{p *} \exp \left(2 \mathbf{E}_{\text {old }}^{e l}\right)\right)\right. \\
& \approx \frac{1}{2} \ln \left(\mathbf{g}_{o}^{-1} \mathbf{F}^{p *} \mathbf{h}\right)+\mathbf{g}_{o}^{-1} \mathbf{F}^{p *}\left(\mathbf{E}_{\text {old }}^{e l}\right)
\end{aligned}
$$

here $\mathbf{E}_{\text {old }}^{e l}=\frac{1}{2} \ln \left(\mathbf{h}^{-1} \mathbf{F}^{e l *} \mathbf{h}\right)$ as in conventional finite elasticity.

\subsection{Strain rate tensors}

We define the strain rate tensors as time derivatives of strain tensors. As a result we get the strain rate tensors $\dot{\mathbf{E}}^{\text {tot }}, \dot{\mathbf{E}}^{\text {in }}, \dot{\mathbf{E}}^{e l}$, and $\dot{\mathbf{E}}^{m}$.

On the other hand, there are other rate characteristics for each of the three participating structures, i.e.:

1. The material velocity:

$$
\mathbf{L}_{D}(t, X)=\mathbf{D}(t, X)^{-1} \circ \frac{\partial}{\partial t} \mathbf{D}(t, X) .
$$


This velocity is related to the velocity $\mathbf{L}_{P}=P^{-1} \circ P_{, t}$ introduced in Sec. 2.8 by the relation $\mathbf{L}_{D}=P \circ \mathbf{L}_{P} \circ P^{-1}$. We see now that $\mathbf{D}=\mathbf{D L}_{D}$ and therefore we get the relation between the material strain rate tensor and the material velocity $\mathbf{L}_{D}$ used in $[5,14,20]$ and other papers.

$$
\begin{aligned}
\dot{\mathbf{E}}^{m} & =\frac{1}{2} \mathbf{g}_{o}^{-1} \dot{\mathbf{g}}=\frac{1}{2} \mathbf{g}_{o}^{-1}\left(\dot{D}_{I}^{M} D_{J}^{N} g_{0 M N}+D_{I}^{M} \dot{D}_{J}^{N} g_{o M N}\right) \\
& =\frac{1}{2} \mathbf{g}_{o}^{-1}\left(D_{K}^{M} L_{D}^{K} D_{J}^{N} g_{o} M N+D_{I}^{M} D_{S}^{N} L_{D J}^{S} g_{o} M N\right) \\
& =\frac{1}{2} \mathbf{g}_{o}^{-1}\left(L_{D}^{K}{ }_{I} g_{P K J}+L_{D J}^{S} g_{I S}\right)=\mathbf{g}_{o}^{-1}\left(L_{D}\right)_{(I}^{K} \cdot g_{|K| J)},
\end{aligned}
$$

where in the last formula there is symmetrization by indices $I J$.

2. The total velocity is defined as

$$
\mathbf{V}(X, t)=\frac{\partial}{\partial t} \phi(X, t)
$$

and its gradient is related to the linearized total strain rate tensor $\mathbf{E}_{\text {lin }}^{\text {tot }}=$ $\frac{1}{2} g_{0}^{-1}\left(C(\phi)-g_{0}\right)$ by the relation

$$
\left(g_{0} \dot{\mathbf{E}}_{\text {lin }}^{\text {tot }}\right)_{M N}=h_{i j} V_{(, M}^{i} \phi_{N)}^{j}
$$

Being written in Euler (spatial) coordinates, this relation reduces to the standard one [16].

3. Finally, the velocity of the inelastic deformation,

$$
\mathbf{V}_{1}(X, t)=\frac{\partial}{\partial t} \phi_{1}(X, t),
$$

is related, in a linear approximation, to the (linearized) inelastic strain rate tensor $\dot{\mathbf{E}}_{\text {lin }}^{\text {in }}$ by a relation containing the symmetrized velocity gradient and the material velocity $\mathbf{L}_{D}$. In the calculation that follows, we are using the formula $\frac{\partial}{\partial t} C\left(\phi_{1}\right)=2 h_{i j} V_{1,(M}^{i} \phi_{1, N)}^{j}$ for time derivative of Cauchy-Green tensor of configuration $\phi_{1}$. We have

$$
\begin{aligned}
\dot{\mathbf{E}}_{\text {lin }}^{i n} & =\frac{\partial}{\partial t}\left(\frac{1}{2} \mathbf{g}^{-1}\left(\mathbf{C}\left(\phi_{1}\right)-\mathbf{g}\right)\right)=\frac{1}{2} \frac{\partial}{\partial t}\left(\mathbf{g}^{-1} \cdot \mathbf{C}\left(\phi_{1}\right)\right) \\
= & \frac{1}{2} \mathbf{g}^{-1} \frac{\partial}{\partial t} \mathbf{C}\left(\phi_{1}\right)+\frac{1}{2} \frac{\partial}{\partial t}\left(\mathbf{g}^{-1}\right) \cdot \mathbf{C}\left(\phi_{1}\right) \\
= & \mathbf{g}^{-1}\left[h_{i j} V_{1,(M}^{i} \phi_{1, N)}^{j}\right]+\frac{1}{2} \mathbf{g}^{-1} \dot{\mathbf{g}} \mathbf{g}^{-1} \cdot \mathbf{C}\left(\phi_{1}\right) \\
= & \mathbf{g}^{-1}\left[h_{i j} V_{1,(M}^{i} \phi_{1, N)}^{j}\right]+\mathbf{g}^{-1}\left(\mathbf{g}_{o} \dot{\mathbf{E}}^{m}\right) \mathbf{g}^{-1} \mathbf{C}\left(\phi_{1}\right) \\
& \approx\left(\nabla^{\mathbf{g}_{o}} \mathbf{v}_{1}\right)_{\text {sym }}+\dot{\mathbf{E}}^{m} .
\end{aligned}
$$


Here $\mathbf{v}_{1 M}=\phi_{, M}^{i} h_{i j} \mathbf{V}_{1}^{j}$ is the covariant form of the convective velocity of inelastic configuration $\phi_{1}$; see [29]. In the last approximation, we replaced $\mathbf{g} \approx \mathbf{g}_{o}$ in the first and the second terms and $\mathbf{C}\left(\phi_{1}\right) \approx \mathbf{g}$ in the second term.

\section{Lagrangian, free energy, dissipative potential, and the stress tensors}

Dynamical equations describing the evolution of the system characterized by the variables $\left(\phi, \phi_{1}, \mathbf{g}\right)$ are obtained by combining the canonical (Lagrangian) component and the the dissipative forces.

The Lagrangian in our model is the combination of the kinetic, potential, and internal energy terms:

$$
L=K-\rho_{\text {ref }} u-U(\phi),
$$

where $K=\frac{\rho}{2}|\mathbf{V}|_{h}$ is the density of kinetic energy, $U(\phi)$ is the potential of the volume forces, and $u$ is the internal energy per unit of mass (see Section 4.2).

\subsection{Lagrangian and internal energy}

It is traditional to define the free energy density $\psi$ as a function of the elastic deformation gradient, the temperature $\vartheta$, a material point $X$, and additional internal parameters $\boldsymbol{\alpha}$ (see $[26,30])$.

Dissipative pseudo-potential is, in this approach, the function of rates of deformation gradients and time derivatives of internal variables $\mathcal{D}=\bar{D}\left(\dot{\mathbf{F}}^{e}, \dot{\mathbf{F}}^{p}, \dot{\boldsymbol{\alpha}}, \nabla \vartheta, \nabla \boldsymbol{\alpha}, \vartheta\right)[26,30]$. This allows one to define the total, elastic, and plastic stress tensors and the thermodynamical forces conjugate to the parameters $\boldsymbol{\alpha}$, thus separating different factors in the dissipation inequality (see eq. (10.21.) in [14]).

Comparing the expression for the internal energy (32) with these in $[26,30]$, one sees that the metric $\mathbf{g}$ entering the free energy through the tensor $\mathbf{E}^{m}$ plays here the role of an internal variable $\alpha$ and its Ricci tensor $\operatorname{Ric}(\mathbf{g})$ takes the place of the space gradient $\nabla \boldsymbol{\alpha}[26,30]$. The elastic $\mathbf{E}^{e l}$ (respectively, inelastic $\mathbf{E}^{i n}$ ) strain tensors are direct material analogs of $\boldsymbol{\epsilon}_{e}$ (respectively, of $\boldsymbol{\epsilon}_{p}$ ). Thus it is conceivable to adopt the internal variables approach in searching for the form of the equations governing the behavior of our system. 
We take the Lagrangian in the form

$$
\begin{aligned}
L= & L\left(\rho_{r e f}, \mathbf{V}, \mathbf{E}^{e l}, \mathbf{E}^{i n}, \mathbf{E}^{m}, \operatorname{Ric}(\mathbf{g}), \vartheta, \nabla^{g_{o}} \vartheta\right)=K-\rho_{r e f} u-U(\phi) \\
= & \frac{\rho_{r e f}}{2}|\mathbf{V}|_{h}^{2}+\rho_{r e f}\left[\boldsymbol{\gamma} \cdot \operatorname{Ric}(\mathbf{g})+\mu\left\|\nabla^{g_{o}} \vartheta\right\|_{g}^{2}+f_{0}\left(\mathbf{E}^{i n}, \mathbf{E}^{m}, \vartheta\right)\right. \\
& \left.+f\left(\mathbf{E}^{i n}, \mathbf{E}^{m}, \vartheta ; \mathbf{E}^{e l}\right)\right]-U(\phi) .
\end{aligned}
$$

In this expression $\gamma$ is a constitutive tensor, $U$ is the potential of body forces, $f_{0}$ is the "basic inelastic energy", and $f$ is the strain energy of linear thermoelasticity, i.e., a quadratic function of the elastic strain tensor with coefficients depending on the temperature and the remaining inelastic strains:

$$
f\left(\mathbf{E}^{i n}, \mathbf{E}^{m}, \vartheta ; \mathbf{E}^{e l}\right)=\left[\mathbf{c}_{\mathbf{0}}+\left(\vartheta-\vartheta_{0}\right) \mathbf{c}_{1}\right]: \mathbf{E}^{e l}+\left(\mathbf{e}: \mathbf{E}^{e l}: \mathbf{E}^{e l}\right)
$$

Here $\mathbf{c}_{\mathbf{0}}$ and $\mathbf{c}_{\mathbf{1}}$ are tensors characterizing the interaction of the elastic processes with the inelastic ones and temperature, respectively (for instance, $\mathbf{c}_{\mathbf{1}}$ is the thermal expansion tensor). The tensor $\mathbf{e}$ is the elasticity tensor.

Assuming that the decomposition (23) is valid, substitution of the total strain tensor $\mathbf{E}^{\text {tot }}$ instead of the elastic strain tensor $\mathbf{E}^{e l}$ into the expressions for internal energy and dissipative potential (below) does not change the form of function (39):

$$
\begin{aligned}
f\left(\mathbf{E}^{i n}, \mathbf{E}^{m}, \vartheta ; \mathbf{E}^{t o t}\right)= & f\left(\mathbf{E}^{i n}, \mathbf{E}^{m}, \vartheta ; \mathbf{E}^{t o t}-\mathbf{E}^{i n}-\mathbf{E}^{m}\right) \\
= & {\left.\left[\mathbf{c}_{\mathbf{0}}+\left(\vartheta-\vartheta_{0}\right) \mathbf{c}_{1}\right]:\left(\mathbf{E}^{t o t}-\mathbf{E}^{i n}-\mathbf{E}^{m}\right)\right) } \\
& +\left(\mathbf{e}:\left(\mathbf{E}^{t o t}-\mathbf{E}^{i n}-\mathbf{E}^{m}\right):\left(\mathbf{E}^{t o t}-\mathbf{E}^{i n}-\mathbf{E}^{m}\right)\right) \\
= & +\left(\mathbf{e}:\left(\mathbf{E}^{i n}+\mathbf{E}^{m}\right):\left(\mathbf{E}^{i n}+\mathbf{E}^{m}\right)\right)+\left[\left(\mathbf{c}_{\mathbf{0}}-2 \mathbf{e}:\left(\mathbf{E}^{i n}+\mathbf{E}^{m}\right)\right.\right. \\
& \left.+\left(\vartheta-\vartheta_{0}\right) \mathbf{c}_{1}\right]: \mathbf{E}^{t o t}+\mathbf{e}: \mathbf{E}^{t o t}: \mathbf{E}^{t o t},
\end{aligned}
$$

but changes the tensor $\mathbf{c}_{0}$ and adds a term to the inelastic energy $f_{0}$.

This allows us to replace $\mathbf{E}^{e l}$ by $\mathbf{E}^{\text {tot }}$ in the internal energy, so that we can equivalently use Lagrangian in the form

$$
\begin{aligned}
L= & L\left[\rho_{r e f}, \mathbf{V}, \mathbf{E}^{t o t}, \mathbf{E}^{i n}, \mathbf{E}^{m}, \operatorname{Ric}(\mathbf{g}), \vartheta, \nabla^{g_{o}} \vartheta\right] \\
= & \frac{\rho_{r e f}}{2}|\mathbf{V}|_{h}^{2}+\rho_{r e f}\left[\gamma \cdot \operatorname{Ric}(\mathbf{g})+\mu\left\|\nabla^{g_{o}} \vartheta\right\|_{g}^{2}+f_{0}\left(\mathbf{E}^{i n}, \mathbf{E}^{m}, \vartheta\right)\right. \\
& \left.+f\left(\mathbf{E}^{i n}, \mathbf{E}^{m}, \vartheta ; \mathbf{E}^{t o t}\right)\right]-U(\phi) .
\end{aligned}
$$

The strain energy here has the form (40) where $\mathbf{E}^{e l}$ is replaced by $\mathbf{E}^{\text {tot }}$. 
In the dynamic equations, it is more convenient to use $\mathbf{E}^{\text {tot }}$ since $\phi$ is a geometrically explicit and observable quantity while in the dissipative inequality it is more convenient to use $\mathbf{E}^{e l}$ because it allows one to separate inputs of different processes into the entropy production.

\subsection{Free energy}

The free energy is defined, as usual, by the equality

$$
\psi=u-s \theta
$$

where $s$ is the specific entropy.

\subsection{Dissipative potential}

The dissipative (pseudo) potential is chosen to be a function of the following variables:

$$
\mathcal{D}=\mathcal{D}\left(\dot{\mathbf{E}}^{i n}, \dot{\mathbf{E}}^{m} ; \mathbf{E}^{m}, \vartheta\right) .
$$

We include $\dot{\mathbf{E}}^{\text {in }}$ together with $\dot{\mathbf{g}}$ to emphasize the difference between the kinetic energy related with $\phi_{t}^{\text {tot }}$ and the inelastic strain rate $\dot{\mathbf{E}}^{\text {in }}$ participating in the process of viscous dissipation; see [31].

\subsection{Evolution equations}

Introducing the action for the material in a domain $G \subset M$, corresponding to the Lagrangian $L$,

$$
A\left(\phi, \phi_{1}, \mathbf{g}\right)=\int_{G} L d v_{g},
$$

we will write down the equations of evolution for the system characterized by the dynamical variables $\phi, \phi_{1}, \mathbf{g}$ :

1. Equilibrium equation:

$$
\frac{\delta A}{\delta \phi}=0 .
$$

Since the total deformation $\phi$ enters only through the elastic strain tensor $\mathbf{E}^{e l}$, this equation is, essentially, the equilibrium equation of elasticity 
theory. If the strain energy is chosen in the form (40), this equation takes the conventional form of the elasticity equilibrium (Euler) equation with the elastic moduli depending on the material point and temperature (see Eq. (40)):

$$
\frac{1}{\sqrt{|g|}} \frac{\partial\left(\rho_{r e f} \sqrt{|g|} h_{i j} V^{j}\right)}{\partial t}-\mathrm{P}_{i ; I}^{I}=v_{i}(\phi(X)) .
$$

Here $\boldsymbol{v}=-d U(\phi)$ is the 1 -form of the body forces and $\mathrm{P}_{i}^{I}$ is the elastic first Piola-Kirchhoff stress tensor (see Section 5.5 below). Covariant derivative is taken with respect to the material metric $g$.

2. Equation of plastic deformation:

$$
\frac{\delta A}{\delta \phi_{1}^{i}}=-\frac{\delta \mathbf{D}}{\delta \dot{\phi}_{1}^{i}}
$$

Notice that in difference to the usual form of this equation [26,30], we take the variation of dissipative potential by the derivative $\dot{\phi}_{1}$ of internal variable $\phi_{1}$ rather than the partial derivative. This is necessary due to the fact that $\phi_{1}$ enters Lagrangian through its spatial gradient.

To clarify the form of this equation, we notice that

$$
\begin{aligned}
\frac{\delta A}{\delta \phi_{1}^{i}} & =-\frac{1}{\sqrt{|g|}} \partial_{X^{I}}\left[\frac{\partial u}{\partial \phi_{1, I}^{i}} \sqrt{|g|}\right]=-\operatorname{div}_{g}\left(\mathrm{P}_{1}^{I} i\right)=-\left[T_{M}^{i n} \frac{\partial E_{N}^{i n} M}{\partial \phi_{1, I}^{i}}\right]_{; I} \\
& =-\left[T_{M}^{i n}{ }^{N} g^{M K} h_{i j}\left(\phi_{, N}^{j} \delta_{K}^{I}+\phi_{K}^{j} \delta_{N}^{I}\right)\right]_{; I} .
\end{aligned}
$$

Here $\mathrm{P}_{1 i}^{I}$ is the first Piola-Kirchhoff stress tensor density of inelastic configuration $\phi_{1}$; see below. Last equality is due to the fact that $\frac{\partial E_{\operatorname{lin} N}^{i n} N}{\partial \phi_{1, I}^{i}}=$ $g^{M K} h_{i j}\left(\phi_{, N}^{j} \delta_{K}^{I}+\phi_{K}^{j} \delta_{N}^{I}\right)$.

On the other hand,

$$
\begin{aligned}
\frac{\delta \mathbf{D}}{\delta \dot{\phi}_{1}^{i}} & =-\partial_{K}\left[\frac{\partial \mathbf{D}}{\partial \dot{E}_{N}^{i n} M} \frac{\partial \dot{E}_{N}^{i n} M}{\partial \dot{\phi}_{1, K}^{i}}\right] \\
& =-\partial_{K}\left[\frac{\partial \mathbf{D}}{\partial \dot{E}_{N}^{i n} M} \cdot \frac{1}{2} h_{i j}\left(g^{M K} \phi_{1, N}^{i}+g^{M S} \phi_{1, S}^{j} \delta_{N}^{K}\right)\right],
\end{aligned}
$$


where we have used Eq. (36). As a result, Eq. (45) has the form

$$
\operatorname{div}_{g}\left(\mathrm{P}_{1 i}^{I}\right)+\partial_{K}\left[\frac{\partial \mathbf{D}}{\partial \dot{E}_{N}^{i n} M} \cdot \frac{1}{2} h_{i j}\left(g^{M K} \phi_{1, N}^{i}+g^{M S} \phi_{1, S}^{j} \delta_{N}^{K}\right)\right]=0 .
$$

3. Equation of metric evolution:

$$
\frac{\delta A}{\delta \mathbf{g}}=-\frac{\partial \mathbf{D}}{\partial \dot{\mathbf{g}}} .
$$

Here we have used the partial derivatives in the right side of the equation since $\mathbf{E}^{m}=\frac{1}{2} \ln \left(\mathbf{g}_{0}^{-1} \mathbf{g}\right)$ depends on $\mathbf{g}$ but not on its derivatives.

If the free energy depends on the scalar curvature $R(\mathbf{g})$ instead, the full Ricci tensor, Eq. (48), has the form

$$
c \cdot \mathcal{E}(\mathbf{g})^{I J}=-S^{m} I J-\frac{\partial \mathbf{D}}{\partial \dot{\mathbf{E}}^{m}}
$$

where

$$
S^{m I J}=\frac{1}{\sqrt{|\mathbf{g}|}} \frac{\delta}{\delta g_{I J}}\left(\left[\frac{\rho_{r e f}}{2}|\mathbf{V}|_{h}^{2}+\rho_{r e f}\left[\mu\left\|\nabla^{g_{o}} \vartheta\right\|_{g_{o}}^{2}+f_{0}+f\right]-U(\phi)\right] \sqrt{|\mathbf{g}|}\right)
$$

is the canonical energy-momentum tensor including elastic effects, effects of inelastic deformation, and some thermal effects, and $\mathcal{E}(\mathbf{g})^{I J}$ is the Einstein tensor of metric $g$ [32]. If $\mathbf{g}=\mathbf{g}_{o}$ is the reference metric, then this equation is absent ( $\mathbf{g}_{0}$ is fixed).

Remark 11 In the 2-dim elasticity, any metric $g$ in $M$ is Einstein metric, i.e, $R i_{I J}=\frac{R(g)}{2} g_{I J}$. In this case using the scalar curvature $R(g)$ instead of the Ricci tensor in $(38,41)$ does not place any restrictions on the material metric $g$.

\subsection{Stress tensors}

Stress tensors characterizing the material's response to the deformations, heating and other physical processes play a crucial role in the formulation of the evolution equations and dissipative inequalities. In the material (Lagrangian) formulation, there are several stress tensors playing different roles in the dynamical picture. They are related to one another and, through the deformation $\phi$, to the only stress tensor that is usually present in the Euler picture - the Cauchy tensor $\sigma$. Such a plurality of material stress tensors is related to the presence of two material metrics, i.e., $\mathbf{g}_{o}, \mathbf{g}$, used to raise and lower the indices in tensors, and the two different Cauchy metrics $-\mathbf{C}(\phi), \mathbf{C}\left(\phi_{1}\right)$. 
Here we recall the definitions of the most useful stress tensors through the total internal energy $u$ or the strain energy $f$ and provide the formulas relating them to one another $[14,15,33]$.

For the total deformation $\phi$, we introduce three stress tensors defined by the differentiation of internal energy by the deformation gradient $F=\phi_{*}$, Cauchy metric $C(\phi)$, and the strain tensor $E^{\text {tot }}$.

Table 1 Stress tensors defined by total deformation $\phi$.

\begin{tabular}{llrr}
\hline Type & I Piola-Kirchhoff & II Piola-Kirchhoff & Strain dual \\
\hline Tensor & $\mathrm{P}_{i}^{I}=\rho_{\text {ref }} \frac{\partial u}{\partial \phi_{, I}^{i}}$ & $S^{I J}=2 \rho_{\text {ref }} \frac{\partial u}{\partial C(\phi)_{I J}}$ & $T_{J}^{e l}=\rho_{\text {ref }} \frac{\partial u}{\partial E_{I}^{e I J}}$ \\
\hline Relations & $\mathrm{P}_{i}^{I}=J(\phi) \sigma_{i}^{j} \phi_{j}^{-1 I}$ & $\mathrm{P}^{I i}=S^{I K} \phi_{, K}^{i}$ & $T_{J}^{e l I}=S^{I K} C\left(\phi_{1}\right)_{K J}$ \\
\hline
\end{tabular}

The formula relating $S$ and $T^{e l}$ is obtained in the assumption of linear approximation $C(\phi)=C\left(\phi_{1}\right)+2 C\left(\phi_{1}\right) E^{e l}$; see Section 4.1. $J(\phi)$ here is the Jacobian of the total deformation $\phi$ calculated with respect to the metrics $\mathbf{h}$ and $\mathbf{g}_{o}$; see [15], Sec. 2.2. Expression for the tensor $S^{\text {tot } I J}$ in Table 1 is the material Doyle-Erickson formula (see [33]).

For the inelastic deformation $\phi_{1}$, we introduce three stress tensors defined by the differentiation of internal energy by the deformation gradient $F=\phi_{1 *}$, Cauchy metric $C\left(\phi_{1}\right)$, and the strain tensor $E^{\text {in }}$ (see Section 4.1) (Table 2).

Table 2 Stress tensors defined by inelastic deformation $\phi_{1}$.

\begin{tabular}{lllr}
\hline Type & I Piola-Kirchhoff & II Piola-Kirchhoff & Strain dual \\
\hline Tensor & $\mathrm{P}_{1 i}^{I}=\rho_{\text {ref }} \frac{\partial u}{\partial \phi_{1, I}^{i}}$ & $S_{1}^{I J}=2 \rho_{\text {ref }} \frac{\partial u}{\partial C(\phi)_{1 I J}}$ & $T_{J}^{\text {in } I}=\rho_{\text {ref }} \frac{\partial u}{\partial E_{I}^{i n}}$ \\
\hline Relations & $\mathrm{P}_{1 i}^{I}=J\left(\phi_{1}\right) \sigma_{i}^{j} \phi_{j}^{-1 I}$ & $\mathrm{P}_{1}^{I i}=S_{1}^{I K} \phi_{1, K}^{i}$ & $T_{J}^{i n I}=S_{1}^{I K} g_{g_{K J}}$ \\
\hline
\end{tabular}

The relation between $S_{1}$ and $T^{\text {in }}$ is obtained in the assumption of linear approximation $E^{i n}=\frac{1}{2} g^{-1}\left(C\left(\phi_{1}\right)-g\right)$.

For the deformation (evolution) of material metric $g_{0} \rightarrow g_{t}$, there are defined the stress tensors as shown in Table 3.

Here $P_{X}^{I}{ }_{i}$ is the uniformity mapping $P: V \rightarrow T_{X}(X)$ and the internal energy is referered to the reference volume $d_{g_{0}} V,[11]$, Ch. 5, Sec. 5.5. Formula relating $S^{m}$ and $T^{m}$ is obtained in the assumption of linear approximation $E^{m}=\frac{1}{2} g_{0}^{-1}\left(g-g_{0}\right)$. 
Table 3 Stress tensors defined by the material metric.

\begin{tabular}{llcr}
\hline Type & Eshelby stress & Canonical & Strain dual \\
\hline Tensor & $b_{I}^{i}=-\rho_{\text {ref }} \frac{\partial u}{\partial P_{i}^{I}}$ & $S^{m I J}=2 \rho_{\text {ref }} \frac{\partial u}{\partial g_{I J}}$ & $T_{J}^{m I}=\rho_{\text {ref }} \frac{\partial u}{\partial E_{I}^{m J}}$ \\
\hline Relations & & $b_{I}^{i}=S^{m} M P_{M}^{-1}{ }^{i} g_{N I}$ & $T_{J}^{m I}=S^{m I K_{g}} g_{0 J}$ \\
\hline
\end{tabular}

One can define the variant of the Eshelby stress $[11,12,14]$ by $\tilde{b}_{J}^{I}=-\rho_{0} \frac{\partial u}{\partial D_{I}^{J}}$ using the material $(1,1)$-tensor $D$. Its relation to the tensor $b_{I}^{i}$ is given by $\tilde{b}_{J}^{I}=b_{J}^{i} P_{0 i}^{-1 I}$.

Notice also that the canonical stress tensor $S^{m}$ is the direct analog of the spatial part of the energy-momentum tensor of the gravity theory [32].

Remark 12 It is instructive to compare our definition of the elastic first PiolaKirchhoff tensor with its definition as the difference (cf. [4], Ch. 10)

$$
T_{i}^{e l I}=T_{i}^{I}-\phi_{2, i}^{j} T_{j}^{i n I},
$$

where $\phi_{2}=\phi \circ \phi_{1}^{-1}$ is the elastic part of total deformation.

\section{Dissipation inequality}

In this section we present the dissipative inequality for the $\left(\phi, \phi_{1}, g\right)$ model.

Below, $\nabla$ means $\nabla^{g_{o}}$. We will adopt here the expression (32) for the internal energy but assume, for simplicity, the quasi-static behavior of the material (i.e., velocity $\mathbf{V}$ is negligible), potential energy $U$ is absent, and internal energy $u$ depends on the scalar curvature $R(\mathbf{g})$ only, instead of on the full Ricci tensor:

$$
\psi=u\left(\mathbf{E}^{e l}, \mathbf{E}^{i n}, \mathbf{E}^{m}, R(\mathbf{g}), \vartheta, \nabla^{g_{o}} \vartheta\right)-s \theta,
$$

with $\mathbf{E}^{m}=\frac{1}{2} \ln \left(\mathbf{g}_{o}^{-1} \mathbf{g}\right)$ and $\mathbf{g}$ playing the role of an internal parameter $\boldsymbol{\alpha}$ (cf. [30]).

We will be using the notations

$$
\tilde{s}=-\frac{\partial \psi}{\partial \vartheta}, \mathbf{A}=\frac{\partial \psi}{\partial \nabla \vartheta},
$$

and the formula for the time derivative of the scalar curvature

$$
\frac{\partial}{\partial t} R(\mathbf{g})=\lim _{\Delta t \rightarrow 0}[R(\mathbf{g})(t+\Delta t)-R(\mathbf{g})(t)]=\mathcal{E}(\mathbf{g}) \cdot \dot{\mathbf{g}},
$$


where $\mathcal{E}(\mathbf{g})=\frac{\delta R(\mathbf{g})}{\delta \mathbf{g}}$ is the Einstein tensor of the material metric $\mathbf{g}$ (see [32], eq. (8.49)).

Calculate now the derivative of the free energy:

$$
\begin{aligned}
\dot{\psi}= & \mathbf{T}^{e l} \cdot \dot{\mathbf{E}}^{e l}+\mathbf{T}^{i n} \cdot \dot{\mathbf{E}}^{i n}+\frac{1}{2} \mathbf{S}^{m} \cdot \dot{\mathbf{g}}+\mathcal{E}(\mathbf{g}) \cdot \dot{\mathbf{g}} \\
& -\tilde{s} \cdot \dot{\theta}+\nabla \cdot(\mathbf{A} \dot{\vartheta})-(\nabla \cdot \mathbf{A}) \dot{\vartheta},
\end{aligned}
$$

where the vectorial identity $\nabla \cdot(\mathbf{A} \dot{\vartheta})=(\nabla \cdot \mathbf{A}) \dot{\vartheta}+\mathbf{A} \cdot \nabla \dot{\vartheta}$ was used and where tensors $T^{\text {in }}, T^{e l}, S^{m}$ are as in the Section 5.

Recalling the formula for the variation

$$
\frac{\delta \psi}{\delta \mathbf{g}}=\frac{\partial \psi}{\partial \mathbf{g}}-\nabla \cdot \frac{\partial \psi}{\partial \nabla \mathbf{g}_{o} \mathbf{g}}
$$

and using the notation $\mathcal{A}=\frac{\delta \psi}{\delta \mathbf{g}}$, we find

$$
\left[\frac{1}{2} \mathbf{S}^{m}+\mathcal{E}(\mathbf{g})\right] \cdot \dot{\mathbf{g}}=\frac{\delta \psi}{\delta \mathbf{g}} \cdot \dot{\mathbf{g}}=\mathcal{A}(\mathbf{g}) \cdot \dot{\mathbf{g}} .
$$

Then the time derivative of the free energy takes the form

$$
\dot{\psi}=\mathbf{T}^{e l} \cdot \dot{\mathbf{E}}^{e l}+\mathbf{T}^{i n} \cdot \dot{\mathbf{E}}^{i n}+\mathcal{A}(\mathbf{g}) \cdot \dot{\mathbf{g}}-\tilde{s} \cdot \dot{\vartheta}-\nabla \cdot(\mathbf{A}) \dot{\vartheta}+\nabla \cdot(\mathbf{A} \dot{\vartheta}) .
$$

Recall now the Gibbs inequality for a thermodynamical system with internal parameter $\boldsymbol{\alpha}$ (here $\boldsymbol{\alpha}=\mathbf{g}$ ); see [30]:

$$
-(\dot{\psi}+s \dot{\theta})+p_{i}+\nabla \cdot(\theta \mathbf{k})-(s \cdot \nabla) \theta \geq 0 .
$$

Here

$$
p_{i}=\mathbf{T} \cdot \dot{\mathbf{E}}^{t o t}
$$

is the power of the internal work, stress tensor $\mathbf{T}$ will be specified below, and $\mathbf{k}$ is the extra entropy flux density assumed to include contributions from the flux of the internal variables.

Substituting the expression (56) for $\dot{\psi}$ into the Gibbs inequality (57), we get

$$
\begin{aligned}
& -\mathbf{T}^{e l} \cdot \dot{\mathbf{E}}^{e l}-\mathbf{T}^{i n} \cdot \dot{\mathbf{E}}^{i n}-\mathcal{A}(g) \cdot \dot{\mathbf{g}}+\tilde{s} \cdot \dot{\vartheta}+\nabla \cdot(\mathbf{A} \dot{\vartheta})-, \\
& \nabla \cdot(\mathbf{A} \dot{\vartheta}-s \dot{\theta})+p_{i}+\nabla \cdot(\vartheta \mathbf{k})-(s \cdot \nabla) \vartheta \geq 0 .
\end{aligned}
$$

In the special case of when one uses the linearized definitions of strain tensors (see Section 4.1) and the commutativity condition that allows us to write the 
total strain rate $\dot{E}^{\text {tot }}$ in the form (23) is fulfilled, the previous inequality takes the form

$$
\begin{aligned}
& \left(\mathbf{T}\left(1+2 E^{m}\right)\left(1+2 E^{i n}\right)-\mathbf{T}^{e l}\right) \cdot \dot{\mathbf{E}}^{e l} \\
& \quad+\left(\mathbf{T}\left(1+2 E^{m}\right)\left(1+2 E^{e l}\right)-\mathbf{T}^{i n}\right) \cdot \dot{\mathbf{E}}^{i n} \\
& \quad+\mathbf{T}\left(1+2 E^{e l}\right)\left(1+2 E^{i n}\right) \cdot \dot{\mathbf{E}}^{m}-\mathcal{A}(\mathbf{g}) \cdot \dot{\mathbf{g}} \\
& \quad+(s-\tilde{s}+\nabla \cdot \mathbf{A}) \dot{\vartheta}+\nabla \cdot(\theta \mathbf{k}-\mathbf{A} \dot{\theta})-(s \cdot \nabla) \theta \geq 0 .
\end{aligned}
$$

Now we use the fact that the derivatives $\dot{E}^{e l}, \dot{\vartheta}$ are controllable variables and can take arbitrary positive and negative values and, therefore, their coefficients should be equal to zero [30]. Thus we obtain the relations

$$
\mathbf{T}=\mathbf{T}^{e l}\left(1+2 E^{i n}\right)^{-1}\left(1+2 E^{m}\right)^{-1}
$$

and

$$
s=-(\tilde{s}-\nabla \cdot \mathbf{A})=-\left(\frac{\partial \psi}{\partial \vartheta}-\nabla \cdot \frac{\partial \psi}{\partial \nabla \vartheta}\right)=-\frac{\delta \psi}{\delta \vartheta} .
$$

Assuming for $\mathbf{k}$ the prescription

$$
\mathbf{k}=\vartheta^{-1} \mathbf{A} \dot{\vartheta}=\vartheta^{-1} \frac{\partial \psi}{\partial \nabla \vartheta} \dot{\vartheta},
$$

the reduced dissipation inequality is obtained in the form

$$
\begin{aligned}
& {\left[\mathbf{T}^{e l}\left(1+2 E^{i n}\right)^{-1}\left(1+2 E^{e l}\right)-\mathbf{T}^{i n}\right] \cdot \dot{\mathbf{E}}^{i n}} \\
& \quad+\left[\mathbf{T}^{e l}\left(1+2 E^{i n}\right)^{-1}\left(1+2 E^{m}\right)^{-1}\left(1+2 E^{e l}\right)\left(1+2 E^{i n}\right)\right. \\
& \left.\quad-2 \mathcal{A}(\mathbf{g}) g_{0}\right] \cdot \dot{\mathbf{E}}^{m}-(s \cdot \nabla) \vartheta \geq 0,
\end{aligned}
$$

where we have used the expression $\dot{\mathbf{E}}^{m}=\frac{1}{2}\left(g_{0}^{-1} \dot{g}-I\right)=\frac{1}{2} g_{0}^{-1} \dot{g}$ for the linearized metric strain tensor $E^{m}=\frac{1}{2}\left(g_{0}^{-1} g-I\right)$.

Dissipation inequality (64) is satisfied if one requests the independent fulfillment of the stronger conditions - two intrinsic dissipation inequalities:

$$
\left\{\begin{array}{l}
{\left[\mathbf{T}^{e l}\left(1+2 E^{i n}\right)^{-1}\left(1+2 E^{e l}\right)-\mathbf{T}^{i n}\right] \cdot \dot{\mathbf{E}}^{i n} \geq 0,} \\
{\left[T^{e l}\left(1+2 E^{i n}\right)^{-1}\left(1+2 E^{m}\right)^{-1}\left(1+2 E^{e l}\right)\left(1+2 E^{i n}\right)-2 \mathcal{A}(\mathbf{g}) g_{0}\right] \cdot \dot{\mathbf{E}}^{m} \geq 0,}
\end{array}\right.
$$

and the thermal dissipation inequality:

$$
-(s \cdot \nabla) \theta \geq 0 .
$$


Using the relation between the tensor $S^{m}$ and $T^{m}$ presented in Table 3, we can rewrite second inequality in the form

$$
\left[T^{e l}\left(1+2 E^{i n}\right)^{-1}\left(1+2 E^{m}\right)^{-1}\left(1+2 E^{e l}\right)\left(1+2 E^{i n}\right)-T^{m}-2 \mathcal{E}(g) g_{0}\right] \cdot \dot{\mathbf{E}}^{m} \geq 0 .
$$

Comparing inequalities (65-67) with similar dissipative inequalities in [30, 31 , we see that the coefficient of $\dot{E}^{\text {in }}$ (respectively, $\dot{E}^{m}$ ) can be interpreted as the effective stress tensor for integrable inelastic deformation (respectively, for evolution of the uniform structure). Such modifications of the stress tensors are customary in studying the entropy production by a combination of interrelated elastic and inelastic processes; cf. [14], Ch. 10.

\subsection{Yield condition from dissipative inequality}

If all three strain tensors in Eq. (67) are small (in comparison with the unit tensor), the inequalities (67) take the (approximate) form

$$
\left\{\begin{array}{l}
{\left[\mathbf{T}^{e l}-\mathbf{T}^{i n}\right] \cdot \dot{\mathbf{E}}^{i n} \geqq 0,} \\
{\left[T^{e l}-T^{m}-2 \mathcal{E}(g) g_{0}\right] \cdot \dot{\mathbf{E}}^{m} \geqq 0 .}
\end{array}\right.
$$

These inequalities can be interpreted as the yield conditions determining when the corresponding type of inelastic evolution (plastic integrable: $\dot{\phi}_{1} \neq 0$ and/or material metric $\dot{g}_{t} \neq 0$, respectively) may proceed. In each case, the elastic stress $\mathbf{T}^{e l}$ should be large enough to overcome the barrier necessary for initiation of the corresponding process.

This form of yield condition is similar to the condition for the plastic deformation to proceed obtained from the Drucker postulate; see [34], Sec. 8.11, inequality (8.85).

Solutions of evolution equations (45) and (48) describe also the evolution of stress tensors $T^{i n}, T^{m}$. Therefore, the conditions (68) for elastic stress $T^{e l}$ evolves in time. This evolution can be related to the hardening processes during an elasto-plastic deformation of materials.

Consider, for instance, a homogeneous isotropic case. Let $Q_{I J}$ be a symmetric $(0,2)$-tensor. The evolution in the direction of this tensor, i.e., the evolution for which $\dot{E}_{I J}^{i n}=\lambda(t) Q_{I J}, \lambda(t)>0$, may proceed only if the difference $\left(T_{I J}^{e l}-T_{I J}^{i n}\right)$ is such that $\operatorname{Tr}_{g}\left(\left(T_{I J}^{e l}-T_{I J}^{i n}\right) Q^{I J}\right) \geqq 0$, i.e., if this difference is positive in the direction of tensor $Q_{I J}$.

Leaving further study and comparison of these conditions with the usual yield criteria $[34,35]$ for future work, we notice only that the conditions (68) are 
anisotropic by nature and might possibly provide a useful supplement to the usual criteria in essentially anisotropic situations.

\section{Conclusions}

In this work we analyzed the relation between the Bilby-Kröner-Lee multiplicative decomposition $\mathbf{F}=\mathbf{F}^{e} \mathbf{F}^{p}$ of the total deformation gradient into elastic and plastic factors [2-4,12] and the theory of uniform materials [7-9]. We prove that the Bilby-Kröner-Lee multiplicative decomposition is equivalent to the uniform material model with two deformation mappings, i.e., the total $\phi$ and the inelastic $\phi_{1}$ deformations together with the uniformity structure. Uniformity enters through the $(1,1)$-tensor field $\mathbf{D}$ in the material manifold $M$ or through the material metric g. We introduced the total, the elastic, and the inelastic strain tensors characterizing different types of the geometrical evolution of the material. After discussing the relations between these strain tensors and the deformation gradients $\mathbf{F}^{e}$ and $\mathbf{F}^{p}$, we chose the form of the internal energy (38) and of the dissipative potential (42) for the materials modeled by the triple $\left(\phi, \phi_{1}, \mathbf{g}\right)$. The evolution equations were written down for all dynamical variables $\left(\phi, \phi_{1}, \mathbf{g}\right)$. We discussed different types of stress tensors that naturally enter the scheme of our work. Finally, we wrote down the dissipative inequalities for the materials of $\left(\phi, \phi_{1}, \mathbf{g}\right)$-type, where the terms corresponding to the different types of dissipative processes are separated.

Further research along the lines indicated in this paper seems to be in order. First, one should compare our results with those obtained by Maugin in a different framework $[14,30]$. Second, in the continuation of this work we will study the evolution equations (43), (45), (48), obtain the energy balance law, and the heat equation that follows from it along the lines of [14]. Third, some special cases and examples will be considered.

\section{Acknowledgements}

The authors would like to thank Professor M. Elzanowski who made the manuscript of the book [11] available to them before publication and for valuable advice during the preparation of the manuscript.

\section{Appendix}

In this Appendix we present the calculation of the total strain rate $\dot{E}^{\text {tot }}$ that was used in Section 6. 
From the formula (23) for the linearized definition of strain tensors, we get

$$
E^{t o t}=g_{0}^{-1} C\left(\phi_{1}\right) E^{e l}+g_{0}^{-1} g E^{i n}+E^{m} .
$$

Taking derivative, we get

$$
\dot{E}^{t o t}=g_{0}^{-1} C\left(\dot{\phi}_{1}\right) E^{e l}+g_{0}^{-1} C\left(\phi_{1}\right) \dot{E}^{e l}+g_{0}^{-1} \dot{g} E^{i n}+g_{0}^{-1} g \dot{E}^{i n}+\dot{E}^{m} .
$$

From the definition of linearized $E^{i n}=\frac{1}{2} g^{-1}\left(C\left(\phi_{1}\right)-g\right)$, we get $C\left(\phi_{1}\right)=$ $g+2 g E^{i n}$ and, therefore, $\dot{C}\left(\phi_{1}\right)=\dot{g}+2 \dot{g} E^{i n}+2 g \dot{E}^{i n}$. As a result,

$$
g_{0}^{-1} C\left(\dot{\phi}_{1}\right)=g_{0}^{-1}\left(\dot{g}+2 \dot{g} E^{i n}+2 g \dot{E}^{i n}\right)=2 \dot{E}^{m}+4 \dot{E}^{m} E^{i n}+2\left(1+2 E^{m}\right) \dot{E}^{i n},
$$

where we have used $g_{0}^{-1} g=1+2 E^{m}$.

In the second term in Eq. (69), $g_{0}^{-1} C\left(\phi_{1}\right)=g_{0}^{-1} g g^{-1} C\left(\phi_{1}\right)=\left(1+2 E^{m}\right)(1+$ $\left.2 E^{i n}\right)$, in the third one, $g_{0}^{-1} \dot{g}=2 \dot{E}^{m}$. Substituting these expressions into Eq. (69) and collecting coefficients of strain rate tensors, we get

$$
\dot{E}^{t o t}=\left(1+2 E^{m}\right)\left(1+2 E^{i n}\right) \dot{E}^{e l}+\left(1+2 E^{m}\right) \dot{E}^{i n}\left(1+2 E^{e l}\right)+\dot{E}^{m}\left(1+2 E^{e l}\right)\left(1+2 E^{i n}\right) .
$$

In a case where strain tensors participating in the second and third terms of the last formula commute with the corresponding strain rate tensor, we get

$$
\dot{E}^{t o t}=\left(1+2 E^{m}\right)\left(1+2 E^{i n}\right) \dot{E}^{e l}+\left(1+2 E^{m}\right)\left(1+2 E^{e l}\right) \dot{E}^{i n}+\left(1+2 E^{e l}\right)\left(1+2 E^{i n}\right) \dot{E}^{m} .
$$

\section{References}

[1] Bilby, B., Gardner, L., Stroh, A., Continuous distribution of dislocations and the theory of plasticity, in: Proc. XIth ICTAM, Vol. VIII, 35-44, Presse de 1'Universite de Bruxelles, Bruxelles, 1957.

[2] Kröner, E., Kontinuumstheorie der Versetzungen und Eigenspannungen, Springer, Berlin, 1958.

[3] Lee, E., Elastic plastic deformation at finite strain, ASME, Trans. J. Appl. Mech., 54 (1969), 1-6.

[4] Maugin, G.A., The Thermomechanics of Plasticity and Fracture, CUP, 1992.

[5] Maugin, G.A., Epstein, M., Geometric Material Structure of Elastoplasticity, Int. J. Plasticity, 14 (1998), 09-115.

[6] Cleja-Tigoiu, S., Role of the non-riemannian plastic connection in finite elastoplasticity with continuous distribution of dislocations, in: "Mechanics of Material Forces", Eds. P. Steinmann, G. Maugin, pp. 141-148, Springer, Berlin, 2005.

[7] Noll, W., Materially uniform simple bodies with inhomogeneities, Arch. Rat. Mech. Anal., 27 (1967), 1-32. 
[8] Truesdell, C., Wang, C.C., Introduction to Rational Elasticity, Noordhoff, Groningen, 1973.

[9] Wang, C.C., On the geometrical structure of simple bodies, or mathematical formulation for the theory of continuous distributions of dislocations, Arch. Rat. Mech. Anal., 27 (1967), 33-94.

[10] Elzanowski, M., Epstein, M., Geometric Characterization of Hyperelastic Uniformity, Arch. Rat. Mech. Anal., 88 (1985), 347-357.

[11] Epstein, M., Elzanowski, M., Material Inhomogeneitites and their Evolution, Springer, Berlin, 2007.

[12] Epstein, M., Maugin, G.A., On the geometrical material structure of anelasticity, Acta Mechanica, 115 (1996), 119-131.

[13] Preston, S., Notes on the geometry and mechanics of uniform materials, manuscript, 2005.

[14] Maugin, G.A., Material Inhomogeneities in Elasticity, Chapman and Hall, New York, 1993.

[15] Marsden, J., Hughes, T., Mathematical Foundations of Elasticity, Dover, New York, 1983

[16] Truesdell, C., Noll, W., The Non-Linear Field Theories of Mechanics, 2nd ed., Springer, Berlin, 1992.

[17] Kobayashi, S., Nomizu, K., Foundations of Differential Geometry, Wiley (Interscience), New York, Vol. I, 1963; Vol. II, 1969.

[18] Cohen, H., Epstein, M., Remarks on Uniformity in Hyperelastic Materials, Int. J. Solid. Struct., 20 (1984), 233-243.

[19] Cartan, E., Elie Cartan-Albert Einstein: Letters on Absolute Parallelism, 1929-1932, Princeton University Press, Princeton, 1979.

[20] Binz, E., Elzanowski, M., Another look at the evolution of material structures, Math. Mech. Solids, 7 (2002), 203-214.

[21] Ciancio, V., Dolfin, M., Francaviglia, M., Localization of deformations in finite elasticity, Technische Mechanik, 22 (2002), 111-117.

[22] Sedov, L., Berdichevski, N., A dynamical theory of dislocations, Appl. Math. Mech. (P.M.M.), 31 (1967), 6.

[23] Sidoroff, F., The geometrical concept of intermediate configuration and elasto-plastic finite strain, Arch. Mech. (Poland), 25 (1973), 299-308.

[24] Ciancio, V., Francaviglia, M., Rogolino, P., Elastic-plastic deformation of a single crystal in a geometrical theory of thermodynamical spaces with internal variables, Balkan J. Geometry Appl., 9 (2004), 1-12.

[25] Lee, E., Agah-Tehrani, A., The fusion of physical and continuum-mechanical concepts in the formulation of constitutive relations for elastic-plastic materials, in: Non-Classical Continuum Mechanics, Eds. R. Knops, A. Lacey, pp. 244-259, CUP, Cambridge, 1987.

[26] Maugin, G.A., Muschik, W., Thermodynamics with Internal Variables, J. NonEquilib. Thermodyn. I, 19 (1994), 217-249; II, 19 (1994), 250-289.

[27] Muller, I., Thermodynamics, Pitman, New York, 1985.

[28] Muschik, W., Aspects of Non-Equilibrium Thermodynamics, World Scientific, Singapore, 1990. 
[29] Simo, J., Marsden, J., Krishnaprasad, P., The Hamiltonian Structure of Nonlinear Elasticity, Arch. Rat. Mech. Anal., 104 (1988), 125-183.

[30] Maugin, G.A., Internal Variables and Dissipative Structures, J. Non-Equilib. Thermodyn., 15 (1990), 173-192.

[31] Maugin, G.A., The Thermomechanics of Nonlinear Irreversible Behavior, World Scientific, Singapore, 1999.

[32] Misner, C.W., Thorne, K.S., Wheeler, J.A., Gravitation, Sec. 8.7, Box 8.6., W.H. Freeman, New York, 1973.

[33] Simo, J.C., Marsden, J., On the rotated stress tensor and the material version of the Doyle-Ericksen formula, Arch. Rat. Mech. Anal., 86 (1984), 213-231.

[34] Shames, I., Cozarelli, F., Elastic and Plastic Stress Analysis, pp. 284-288, Prentice Hall, Englewood Cliffs, 1992.

[35] Freudenthal, A., Geringer, H., The mathematical theories of the inelastic continuum, in: Handbuch der Physik, Band VI, Elastizität und Plastizität, Ed. S. Flügge, pp. 229-433, pp. 281-283, Springer, Berlin, 1958.

Paper received: $\quad$ 2007-05-07

Paper accepted: 2008-02-14 